\title{
Normalizers inside amalgamated free product von Neumann algebras
}

\author{
By StefaAn VAes 1 ]
}

Publications of the Research Institute for Mathematical Sciences 50 (2014), 695-721.

\begin{abstract}
Recently, Adrian Ioana proved that all crossed products $L^{\infty}(X) \rtimes\left(\Gamma_{1} * \Gamma_{2}\right)$ by free ergodic probability measure preserving actions of a nontrivial free product group $\Gamma_{1} * \Gamma_{2}$ have a unique Cartan subalgebra up to unitary conjugacy. Ioana deduced this result from a more general dichotomy theorem on the normalizer $\mathcal{N}_{M}(A)^{\prime \prime}$ of an amenable subalgebra $A$ of an amalgamated free product von Neumann algebra $M=M_{1} *_{B} M_{2}$. We improve this dichotomy theorem by removing the spectral gap assumptions and obtain in particular a simpler proof for the uniqueness of the Cartan subalgebra in $L^{\infty}(X) \rtimes\left(\Gamma_{1} * \Gamma_{2}\right)$.
\end{abstract}

\section{Introduction and main results}

All free ergodic nonsingular group actions $\Gamma \curvearrowright(X, \mu)$ on standard probability spaces give rise to a crossed product von Neumann algebra $L^{\infty}(X) \rtimes \Gamma$, in which $L^{\infty}(X)$ is a Cartan subalgebra. More generally, Cartan subalgebras arise as $L^{\infty}(X) \subset L(\mathcal{R})$ where $\mathcal{R}$ is a countable nonsingular Borel equivalence relation on $(X, \mu)$. One of the main questions in the classification of these von Neumann algebras $L^{\infty}(X) \rtimes \Gamma$ and $L(\mathcal{R})$ is whether or not $L^{\infty}(X)$ is their unique Cartan subalgebra up to unitary conjugacy. Indeed, if uniqueness holds, the classification problem is reduced to classifying the underlying (orbit) equivalence relations.

Within Popa's deformation/rigidity theory, there has been a lot of recent progress on the uniqueness of Cartan subalgebras in $\mathrm{II}_{1}$ factors, starting with OP07 where it was shown that all crossed products $L^{\infty}(X) \rtimes \mathbb{F}_{n}$ by free ergodic probability measure preserving (pmp) profinite actions of the free groups $\mathbb{F}_{n}$ have a unique Cartan subalgebra. Note that this provided the first uniqueness theorem for Cartan subalgebras up to unitary conjugacy. The result of [OP07] was gradually extended to profinite actions of larger classes of groups $\Gamma$ in [OP08, CS11, CSU11], but all relied on profiniteness of the action and weak amenability of the group $\Gamma$. At the same time, it was conjectured that crossed products $L^{\infty}(X) \rtimes \mathbb{F}_{n}$ by actions of the free groups could have a unique Cartan subalgebra without any profiniteness assumptions on $\mathbb{F}_{n} \curvearrowright(X, \mu)$.

In a joint work with Popa in [PV11, [PV12], we solved this conjecture and proved that the free groups $\Gamma=\mathbb{F}_{n}$ and all nonelementary hyperbolic groups $\Gamma$ are $\mathcal{C}$-rigid (Cartan-rigid), i.e. for every free ergodic pmp action $\Gamma \curvearrowright(X, \mu)$, the $\mathrm{II}_{1}$ factor $L^{\infty}(X) \rtimes \Gamma$ has a unique Cartan subalgebra up to unitary conjugacy. We obtained this result as a consequence of a general dichotomy theorem about normalizers of amenable subalgebras in crossed product von Neumann algebras $N \rtimes \Gamma$, arising from trace preserving actions of such groups $\Gamma$ on arbitrary tracial $(N, \tau)$.

Then in [Io12, the general dichotomy result of [PV11] has been exploited to establish $\mathcal{C}$-rigidity for arbitrary nontrivial free products $\Gamma=\Gamma_{1} * \Gamma_{2}$ and large classes of amalgamated free products $\Gamma=\Gamma_{1} *_{\Sigma} \Gamma_{2}$. This provided in particular the first non weakly amenable $\mathcal{C}$-rigid groups. The main idea of [Io12] is to use the free malleable deformation from [IPP05] of a crossed product

\footnotetext{
${ }^{1}$ KU Leuven, Department of Mathematics, Leuven (Belgium), stefaan.vaes@wis.kuleuven.be Supported by ERC Starting Grant VNALG-200749, Research Programme G.0639.11 of the Research Foundation - Flanders (FWO) and KU Leuven BOF research grant OT/08/032.
} 
$B \rtimes\left(\Gamma_{1} * \Gamma_{2}\right)$, providing a 1-parameter family of embeddings $\theta_{t}: B \rtimes\left(\Gamma_{1} * \Gamma_{2}\right) \rightarrow N \rtimes \mathbb{F}_{2}$ into some crossed product by the free group $\mathbb{F}_{2}$. Then the main result of [PV11] is applied to this crossed product $N \rtimes \mathbb{F}_{2}$ and a very careful and delicate analysis is needed to "come back" and deduce results about the original crossed product $B \rtimes\left(\Gamma_{1} * \Gamma_{2}\right)$.

The purpose of this article is to give a simpler approach to this "come back" procedure and, at the same time, prove a more general result removing the spectral gap assumptions in [Io12]. As a result, we obtain a simpler proof for the $\mathcal{C}$-rigidity of amalgamated free product groups.

Our method allows to prove a more generic theorem about the normalizer of a subalgebra inside an amalgamated free product of von Neumann algebras, see Theorem A below. This theorem has the advantage to immediately imply a similar result for HNN extensions of von Neumann algebras, see Theorem 4.1. As such we obtain with the same effort $\mathcal{C}$-rigidity for a large class of HNN extensions $\Gamma=\operatorname{HNN}\left(\Gamma_{1}, \Sigma, \theta\right)$, established before in [DI12] using more involved methods.

As we explain below, following the strategy of [HV12], we also prove a uniqueness theorem of Cartan subalgebras in type III factors. This then allows us to give the first examples of type III actions $\Gamma \curvearrowright(X, \mu)$ that are $\mathrm{W}^{*}$-superrigid, i.e. such that the group $\Gamma$ and its action $\Gamma \curvearrowright(X, \mu)$ can be recovered from $L^{\infty}(X) \rtimes \Gamma$, up to induction of actions.

To state the main result of the article, we first recall Popa's theory of intertwining-by-bimodules from [P001, Po03. When $(M, \tau)$ is a tracial von Neumann algebra and $A \subset p M p, B \subset M$ are von Neumann subalgebras, we say that $A \prec_{M} B(A$ embeds into $B$ inside $M)$ if $L^{2}(p M)$ admits a non-zero $A$ - $B$-subbimodule that is finitely generated as a right Hilbert $B$-module. This is "almost" equivalent with the existence of a partial isometry $v \in B$ such that $v A v^{*} \subset B$. By [Po03, Theorem 2.1 and Corollary 2.3], the negation $A \nprec_{M} B$ is equivalent with the existence of a net of unitaries $\left(a_{i}\right)_{i \in I}$ in $\mathcal{U}(A)$ satisfying $\lim _{i}\left\|E_{B}\left(x u_{i} y\right)\right\|_{2}=0$ for all $x, y \in M$.

Also recall from [OP07, Definition 2.2] that $A$ is said to be amenable relative to $B$ inside $M$ if there exists an $A$-central state $\Omega$ on Jones' basic construction von Neumann algebra $p\left\langle M, e_{B}\right\rangle p$ satisfying $\Omega(x)=\tau(x)$ for all $x \in p M p$. When $B$ is amenable, this is equivalent to $A$ being amenable. When $M=D \rtimes \Gamma$ and $\Lambda, \Sigma<\Gamma$ are subgroups, then the relative amenability of $D \rtimes \Lambda$ w.r.t. $D \rtimes \Sigma$ is equivalent with the relative amenability of $\Lambda$ w.r.t. $\Sigma$ inside $\Gamma$, i.e. with the existence of a $\Lambda$-invariant mean on $\Gamma / \Sigma$.

The following is the main result of the article. The same result was proven in [Io12, Theorem 1.6] under the additional assumption that the normalizer $\mathcal{N}_{p M p}(A)=\left\{u \in \mathcal{U}(p M p) \mid u A u^{*}=A\right\}$ of $A$ inside $p M p$ has spectral gap.

Theorem A. Let $M=M_{1} *_{B} M_{2}$ be the amalgamated free product of the tracial von Neumann algebras $\left(M_{i}, \tau\right)$ with common von Neumann subalgebra $B \subset M_{i}$ w.r.t. the unique trace preserving conditional expectations. Let $p \in M$ be a nonzero projection and $A \subset p M p$ a von Neumann subalgebra that is amenable relative to one of the $M_{i}$ inside $M$. Then at least one of the following statements holds.

- $A \prec_{M} B$.

- There is an $i \in\{1,2\}$ such that $\mathcal{N}_{p M p}(A)^{\prime \prime} \prec_{M} M_{i}$.

- We have that $\mathcal{N}_{p M p}(A)^{\prime \prime}$ is amenable relative to $B$ inside $M$.

As in Io12, several uniqueness theorems for Cartan subalgebras can be deduced from Theorem A. This is in particular the case for $\mathrm{II}_{1}$ factors $M=L(\mathcal{R})$ that arise from a countable pmp equivalence relation $\mathcal{R}$ that can be decomposed as a free product $\mathcal{R}=\mathcal{R}_{1} * \mathcal{R}_{2}$ of subequivalence 
relations $\mathcal{R}_{i} \subset \mathcal{R}$. Since we now no longer need to prove the spectral gap assumption, we can directly deduce from Theorem A the following improvement of [Io12, Corollary 1.4] and [BHR12, Theorem 6.3].

Corollary B. Let $\mathcal{R}$ be a countable ergodic pmp equivalence relation on the standard probability space $(X, \mu)$. Assume that $\mathcal{R}=\mathcal{R}_{1} * \mathcal{R}_{2}$ for two subequivalence relations $\mathcal{R}_{i} \subset \mathcal{R}$. Assume that $\left|\mathcal{R}_{1} \cdot x\right| \geq 3$ and $\left|\mathcal{R}_{2} \cdot x\right| \geq 2$ for a.e. $x \in X$. Then $L^{\infty}(X)$ is the unique Cartan subalgebra of $L(\mathcal{R})$ up to unitary conjugacy.

A tracial von Neumann algebra $(M, \tau)$ is called strongly solid if for every diffuse amenable von Neumann subalgebra $A \subset M$, the normalizer $\mathcal{N}_{M}(A)^{\prime \prime}$ is still amenable. For completeness, we also show how to deduce from Theorem A the following stability result for strong solidity under amalgamated free products, slightly improving on [Io12, Theorem 1.8].

For the formulation of the result, recall from [Po03, Section 3] that an inclusion $B \subset\left(M_{1}, \tau\right)$ of tracial von Neumann algebras is called mixing if for every sequence $b_{n} \in B$ with $\left\|b_{n}\right\| \leq 1$ for all $n$ and $b_{n} \rightarrow 0$ weakly, we have that $\lim _{n}\left\|E_{B}\left(x b_{n} y\right)\right\|_{2}=0$ for all $x, y \in M_{1} \ominus B$. Typical examples of mixing inclusions arise as $L(\Sigma) \subset L(\Gamma)$ when $\Sigma<\Gamma$ is a subgroup such that $g \Sigma g^{-1} \cap \Sigma$ is finite for all $g \in \Gamma-\Sigma$, or as $L(\Sigma) \subset B \rtimes \Sigma$ whenever $\Sigma$ acts in a mixing and trace preserving way on $(B, \tau)$.

Corollary C. Let $\left(M_{i}, \tau_{i}\right)$ be strongly solid von Neumann algebras with a common amenable von Neumann subalgebra $B \subset M_{i}$ satisfying $\tau_{1 \mid B}=\tau_{2 \mid B}$. Assume that the inclusion $B \subset M_{1}$ is mixing. Denote by $M=M_{1} *_{B} M_{2}$ the amalgamated free product w.r.t. the unique trace preserving conditional expectations. Then $M$ is strongly solid.

On the level of tracial von Neumann algebras, by [Ue07], amalgamated free products and HNN extensions are one and the same thing, up to amplifications. Therefore, Theorem A has an immediate counterpart for HNN extensions that we formulate as Theorem 4.1 below.

As a consequence, we can then reprove [Io12, Theorem 1.1] and [DI12, Corollary 1.7], showing $\mathcal{C}$-rigidity for amalgamated free product groups, $\mathrm{HNN}$ extensions and their direct products. We refer to Theorem 5.1 for a precise statement.

Finally in Section 8, we use the methods of [HV12 to deduce from Theorem A a uniqueness theorem for Cartan subalgebras in type III factors $L^{\infty}(X) \rtimes \Gamma$ arising from nonsingular free ergodic actions of amalgamated free product groups, see Theorem 8.1, generalizing [BHR12, Theorem D]. As a consequence, we can provide the following first nonsingular actions of type III that are $\mathrm{W}^{*}$-superrigid.

Proposition D. Consider the linear action of $\mathrm{SL}(5, \mathbb{Z})$ on $\mathbb{R}^{5}$ and define the subgroup $\Sigma<$ $\mathrm{SL}(5, \mathbb{Z})$ of matrices $A$ satisfying $A e_{i}=e_{i}$ for $i=1,2$. Put $\Gamma=\mathrm{SL}(5, \mathbb{Z}) *_{\Sigma}(\Sigma \times \mathbb{Z})$ and denote by $\pi: \Gamma \rightarrow \mathrm{SL}(5, \mathbb{Z})$ the natural quotient homomorphism. The diagonal action $\Gamma \curvearrowright \mathbb{R}^{5} / \mathbb{R}_{+} \times[0,1]^{\Gamma}$ given by

$$
g \cdot(x, y)=(\pi(g) \cdot x, g \cdot y),
$$

where $g \cdot y$ is given by the Bernoulli shift, is a nonsingular free ergodic action of type $I_{1} I_{1}$ that is $W^{*}$-superrigid.

This means that for any nonsingular free action $\Gamma^{\prime} \curvearrowright\left(X^{\prime}, \mu^{\prime}\right)$, the following two statements are equivalent.

- $L^{\infty}(X) \rtimes \Gamma \cong L^{\infty}\left(X^{\prime}\right) \rtimes \Gamma^{\prime}$ 
- There exists an embedding of $\Gamma$ into $\Gamma^{\prime}$ such that $\Gamma^{\prime} \curvearrowright X^{\prime}$ is conjugate with the induction of $\Gamma \curvearrowright X$ to a $\Gamma^{\prime}$-action.

To clarify the statement of Proposition D, one should make the following observations. Contrary to the case of probability measure preserving actions, it is not relevant to consider stable isomorphisms, since the type III factor $M=L^{\infty}(X) \rtimes \Gamma$ is isomorphic with $B(H) \bar{\otimes} M$ for every separable Hilbert space $H$. For the same reason, it is unavoidable that $\Gamma^{\prime} \curvearrowright\left(X^{\prime}, \mu^{\prime}\right)$ can be any induction of $\Gamma \curvearrowright(X, \mu)$ and need not be conjugate to $\Gamma \curvearrowright(X, \mu)$ itself.

It is also possible to prove that for $0<\lambda<1$, the analogous action of $\Gamma$ on $\mathbb{R}^{5} / \lambda^{\mathbb{Z}} \times[0,1]^{\Gamma}$ is of type $\mathrm{III}_{\lambda}$ and $\mathrm{W}^{*}$-superrigid in the appropriate sense. The correct formulation is necessarily more intricate because the action is by construction orbit equivalent with the action of $\Gamma \times \mathbb{Z}$ on $\mathbb{R}^{5} \times[0,1]^{\Gamma}$. More generally for a type $\mathrm{III}_{\lambda}$ free ergodic action $\Gamma \curvearrowright(X, \mu)$, there always is a canonically orbit equivalent action $\Gamma \times \mathbb{Z} \curvearrowright\left(X^{\prime}, \mu^{\prime}\right)$ where the $\Gamma$-action preserves the infinite measure $\mu^{\prime}$ and the $\mathbb{Z}$-action scales $\mu^{\prime}$ by powers of $\lambda$.

Acknowledgment. The main part of this work was done at the Institut Henri Poincaré in Paris and I would like to thank the institute for their hospitality.

\section{Preliminaries}

In the proof of our main technical result (Theorem 3.4), we make use of the following criterion for relative amenability due to [OP07] (see also [PV11, Section 2.5]). We copy the formulation of [Io12, Lemma 2.3].

Lemma 2.1 ([OP07, Corollary 2.3]). Let $(M, \tau)$ be a tracial von Neumann algebra and $p \in M$ a nonzero projection. Let $A \subset p M p$ and $B \subset M$ be von Neumann subalgebras. Let $\mathcal{L}$ be any $B$-M-bimodule.

Assume that there exists a net of vectors $\left(\xi_{i}\right)_{i \in I}$ in $p L^{2}(M) \otimes_{B} \mathcal{L}$ with the following properties.

- For every $x \in p M p$, we have $\lim \sup _{i \in I}\left\|x \xi_{i}\right\|_{2} \leq\|x\|_{2}$.

- We have lim $\sup _{i \in I}\left\|\xi_{i}\right\|_{2}>0$.

- For every $a \in \mathcal{U}(A)$, we have that $\lim _{i \in I}\left\|a \xi_{i}-\xi_{i} a\right\|_{2}=0$.

Then there exists a nonzero projection $q$ in the center of $A^{\prime} \cap p M p$ such that $A q$ is amenable relative to $B$ inside $M$.

\section{Key technical theorem}

Throughout this section, we fix an amalgamated free product $M=M_{1} *_{B} M_{2}$ of tracial von Neumann algebras $\left(M_{i}, \tau\right)$ with a common von Neumann subalgebra $B \subset M_{i}$ w.r.t. the unique trace preserving conditional expectations.

\subsection{The malleable deformation of an amalgamated free product}

We recall from [IPP05, Section 2.2] the construction of Popa's malleable deformation of $M$. We denote $G=\mathbb{F}_{2}$, with free generators $a, b \in G$. Write $G_{1}=a^{\mathbb{Z}}$ and $G_{2}=b^{\mathbb{Z}}$. We define $\widetilde{M}=M *_{B}(B \bar{\otimes} L(G))$. Writing $\widetilde{M}_{i}=M_{i} *_{B}\left(B \bar{\otimes} L\left(G_{i}\right)\right)$, we can also view $\widetilde{M}=\widetilde{M}_{1} *_{B} \widetilde{M}_{2}$. Choose self-adjoint elements $h_{j} \in L\left(G_{j}\right)$ with spectrum $[-\pi, \pi]$ such that $u_{a}=\exp \left(i h_{1}\right)$ and 
$u_{b}=\exp \left(i h_{2}\right)$. Define the 1-parameter groups of unitaries $\left(u_{j, t}\right)_{t \in \mathbb{R}}$ in $L\left(G_{j}\right)$ given by $u_{j, t}=$ $\exp \left(i t h_{j}\right)$. We finally define the 1-parameter group of automorphisms $\left(\theta_{t}\right)_{t \in \mathbb{R}}$ of $\widetilde{M}$ given by

$$
\theta_{t}(x)=u_{j, t} x u_{j, t}^{*} \quad \text { for all } x \in \widetilde{M}_{j} .
$$

Note that $\theta_{t}$ is well defined because $u_{j, t} b u_{j, t}^{*}=b$ for all $b \in B$ and $j \in\{1,2\}$.

We define $\mathcal{S}$ as the set of all finite alternating sequences of 1's and 2's, including the empty sequence $\emptyset$. So the elements of $\mathcal{S}$ are the finite sequences $(1,2,1,2, \cdots)$ and $(2,1,2,1, \cdots)$. The length of an alternating sequence $\mathcal{I} \in \mathcal{S}$ is denoted by $|\mathcal{I}|$. For every $\left(i_{1}, \ldots, i_{n}\right) \in \mathcal{S}$, we define $\mathcal{H}_{\left(i_{1}, \ldots, i_{n}\right)} \subset L^{2}(M)$ as the closed linear span of $\left(M_{i_{1}} \ominus B\right) \cdots\left(M_{i_{n}} \ominus B\right)$. By convention, we put $\mathcal{H}_{\emptyset}=L^{2}(B)$. So we have the orthogonal decomposition

$$
L^{2}(M)=\bigoplus_{\mathcal{I} \in \mathcal{S}} \mathcal{H}_{\mathcal{I}}
$$

We denote by $P_{\mathcal{I}}$ the orthogonal projection of $L^{2}(M)$ onto $\mathcal{H}_{\mathcal{I}}$.

Denote $\rho_{t}=|\sin (\pi t) / \pi t|^{2}$. A direct computation gives us that for all $x \in L^{2}(M)$ and all $t \in \mathbb{R}$,

$$
\begin{aligned}
\left\|E_{M}\left(\theta_{t}(x)\right)\right\|_{2}^{2} & =\sum_{\mathcal{I} \in \mathcal{S}} \rho_{t}^{2|\mathcal{I}|}\left\|P_{\mathcal{I}}(x)\right\|_{2}^{2}, \\
\left\|x-\theta_{t}(x)\right\|_{2}^{2} & =\sum_{\mathcal{I} \in \mathcal{S}} 2\left(1-\rho_{t}^{|\mathcal{I}|}\right)\left\|P_{\mathcal{I}}(x)\right\|_{2}^{2}, \\
\left\|\theta_{t}(x)-E_{M}\left(\theta_{t}(x)\right)\right\|_{2}^{2} & =\sum_{\mathcal{I} \in \mathcal{S}}\left(1-\rho_{t}^{2|\mathcal{I}|}\right)\left\|P_{\mathcal{I}}(x)\right\|_{2}^{2} .
\end{aligned}
$$

The last two equalities imply the following transversality property in the sense of [Po06, Lemma 2.1].

$$
\left\|x-\theta_{t}(x)\right\|_{2} \leq \sqrt{2}\left\|\theta_{t}(x)-E_{M}\left(\theta_{t}(x)\right)\right\|_{2} \quad \text { for all } x \in L^{2}(M), t \in \mathbb{R} .
$$

The following is the main technical result of [IPP05]. For a proof of the version as we state it here, we refer to [Ho07, Section 5] and [PV09, Theorem 5.4].

Theorem 3.1 ([IPP05, Theorem 3.1]). Let $p \in M$ be a nonzero projection and $A \subset p M p$ a von Neumann subalgebra. Assume that there exists an $\varepsilon>0$ and a $t>0$ such that $\left\|E_{M}\left(\theta_{t}(a)\right)\right\|_{2} \geq \varepsilon$ for all $a \in \mathcal{U}(A)$. Then at least one of the following statements hold.

- $A \prec_{M} B$.

- There exists an $i \in\{1,2\}$ such that $\mathcal{N}_{p M p}(A)^{\prime \prime} \prec_{M} M_{i}$.

\subsection{The algebra $\widetilde{M}$ as a crossed product with $\mathbb{F}_{2}$ and random walks on $\mathbb{F}_{2}$}

We recall here the fundamental idea of [Io12] to consider $\widetilde{M}$ as a crossed product with the free group $\mathbb{F}_{2}$ and to exploit the spectral gap of random walks on the nonamenable group $G=\mathbb{F}_{2}$. As in [Io06, Remark 4.5] and [Io12, Section 3], we decompose $M=N \rtimes G$, where $N$ is defined as the von Neumann subalgebra of $\widetilde{M}$ generated by $\left\{u_{g} M u_{g}^{*} \mid g \in G\right\}$ and normalized by the unitaries $\left(u_{g}\right)_{g \in G}$. Note that $N$ is the infinite amalgamated free product of the subalgebras $u_{g} M u_{g}^{*}, g \in G$, over the common subalgebra $B$. From this point of view, the action of $G$ on $N$ is the free Bernoulli action. 
For every $i \in\{1,2\}$ and $t \in(0,1)$, we define the maps $\beta_{i, t}: G_{i} \rightarrow \mathbb{R}$ given by

$$
\beta_{i, t}(g)=\tau\left(u_{i, t} u_{g}^{*}\right) \quad \text { for all } g \in G_{i} .
$$

We then denote by $\gamma_{i, t}$ and $\mu_{i, t}$ the probability measures on $G$ given by

$$
\begin{aligned}
\gamma_{i, t}(g) & = \begin{cases}\left|\beta_{i, t}(g)\right|^{2} & \text { if } g \in G_{i}, \\
0 & \text { if } g \notin G_{i},\end{cases} \\
\mu_{i, t} & =\gamma_{i, t} * \gamma_{i, t},
\end{aligned}
$$

where we used the usual convolution product between probability measures on $G$ :

$$
\left(\gamma * \gamma^{\prime}\right)(g)=\sum_{h, k \in G, h k=g} \gamma(h) \gamma^{\prime}(k)
$$

For $\mathcal{I} \in \mathcal{S}$, we finally denote by $\mu_{\mathcal{I}, t}$ the probability measure on $G$ given by

$$
\mu_{\emptyset, t}(g)=\delta_{g, e} \quad \text { and } \quad \mu_{\left(i_{1}, \ldots, i_{n}\right), t}=\mu_{i_{1}, t} * \mu_{i_{2}, t} * \cdots * \mu_{i_{n}, t}
$$

The probability measures $\mu_{\mathcal{I}, t}$ give rise to the Markov operators $T_{\mathcal{I}, t}$ on $\ell^{2}(G)$ given by

$$
T_{\mathcal{I}, t}=\sum_{g \in G} \mu_{\mathcal{I}, t}(g) \lambda_{g}
$$

The support of the probability measures $\gamma_{i, t}$ and $\mu_{i, t}$ equals $G_{i}$. So the support $S$ of the probability measure $\mu_{(1,2), t}$ equals $G_{1} G_{2}$. Since $S S^{-1}$ generates the group $\mathbb{F}_{2}$ and since $\mathbb{F}_{2}$ is nonamenable, it follows from Kesten's criterion (see e.g. [Pi84, Corollary 18.5]) that $\left\|T_{(1,2), t}\right\|<$ 1 for all $t \in(0,1)$. Writing $c_{t}=\left\|T_{(1,2), t}\right\|^{1 / 2}$, we have found numbers $0<c_{t}<1$ such that

$$
\left\|T_{\mathcal{I}, t}\right\| \leq c_{t}^{|\mathcal{I}|-1} \text { for all } \mathcal{I} \in \mathcal{S} \text { and all } 0<t<1
$$

For every $x \in \widetilde{M}$ and $h \in G$, we define $(x)_{h}=E_{N}\left(x u_{h}^{*}\right)$. So with $\|\cdot\|_{2}$-convergence, we have $x=\sum_{h \in G}(x)_{h} u_{h}$. We recall the following result of [Io12].

Lemma 3.2 (โ⿺12, Formula (3.5)]). For all $t \in(0,1), h \in G$ and $x, y \in L^{2}(M)$, we have that

$$
\left\langle\left(\theta_{t}(x)\right)_{h},\left(\theta_{t}(y)\right)_{h}\right\rangle=\sum_{\mathcal{I} \in \mathcal{S}}\left\langle P_{\mathcal{I}}(x), y\right\rangle \mu_{\mathcal{I}, t}(h) .
$$

Also recall from Io12 that Lemma 3.2 yields the following result.

Theorem 3.3 (【Io12, Theorem 3.2]). Let $p \in M$ be a nonzero projection and $A \subset p M p$ a von Neumann subalgebra. Assume that for some $t \in(0,1)$, we have that $\theta_{t}(A) \prec_{\widetilde{M}} N$. Then at least one of the following statements holds.

- $A \prec_{M} B$.

- There exists an $i \in\{1,2\}$ such that $\mathcal{N}_{p M p}(A)^{\prime \prime} \prec_{M} M_{i}$. 
Proof. We prove the theorem by contraposition. So assume that the conclusion fails. By Theorem 3.1. we find a net of unitaries $\left(a_{i}\right)_{i \in I}$ in $\mathcal{U}(A)$ such that for all $s \in(0,1)$, we have that $\lim _{i \in I}\left\|E_{M}\left(\theta_{s}\left(a_{i}\right)\right)\right\|_{2}=0$. We prove that for all $t \in(0,1)$, we have that $\theta_{t}(A) \nprec_{\widetilde{M}} N$. So fix $t \in(0,1)$. It suffices to prove that for all $h \in G$, we have $\lim _{i \in I}\left\|\left(\theta_{t}\left(a_{i}\right)\right)_{h}\right\|_{2}=0$.

Fix $h \in G$ and fix $\varepsilon>0$. Take a large enough integer $n_{0}$ such that $c_{t}^{n_{0}-1}<\varepsilon$. So, for all $\mathcal{I} \in \mathcal{S}$ with $|\mathcal{I}| \geq n_{0}$, we have that $\left\|T_{\mathcal{I}, t}\right\|<\varepsilon$ and, in particular,

$$
\mu_{\mathcal{I}, t}(h)=\left\langle T_{\mathcal{I}, t} \delta_{e}, \delta_{h}\right\rangle<\varepsilon .
$$

Denote by

$$
P_{0}=\sum_{\mathcal{I} \in \mathcal{S},|\mathcal{I}|<n_{0}} P_{\mathcal{I}}
$$

the projection onto the closed linear span of "all words of length $<n_{0}$ ". Using Lemma 3.2 , we get for all $i \in I$ that

$$
\left\|\left(\theta_{t}\left(a_{i}\right)\right)_{h}\right\|_{2}^{2} \leq\left\|P_{0}\left(a_{i}\right)\right\|_{2}^{2}+\varepsilon .
$$

By (3.1), we can take $s>0$ small enough such that $\left\|P_{0}\left(a_{i}\right)\right\|_{2} \leq 2\left\|E_{M}\left(\theta_{s}\left(a_{i}\right)\right)\right\|_{2}$ for all $i \in I$. Since $\lim _{i \in I}\left\|E_{M}\left(\theta_{s}\left(a_{i}\right)\right)\right\|_{2}=0$, it follows that

$$
\limsup _{i \in I}\left\|\left(\theta_{t}\left(a_{i}\right)\right)_{h}\right\|_{2}^{2} \leq \varepsilon .
$$

Since $\varepsilon>0$ is arbitrary, it indeed follows that for all $h \in G$, we have $\lim _{i \in I}\left\|\left(\theta_{t}\left(a_{i}\right)\right)_{h}\right\|_{2}=0$.

\subsection{Relative amenability and the malleable deformation}

The following is our main technical result. The same statement was proven in Io12, Theorem 5.1] under the additional assumption that $A^{\prime} \cap(p M p)^{\omega}=\mathbb{C} 1$ for some free ultrafilter $\omega$, i.e. under the assumption that there are no nontrivial bounded sequences in $p M p$ that asymptotically commute with $A$.

Theorem 3.4. Let $p \in M$ be a nonzero projection and $A \subset p M p$ a von Neumann subalgebra. Assume that for all $t \in(0,1)$, we have that $\theta_{t}(A)$ is amenable relative to $N$ inside $\widetilde{M}$. Then at least one of the following statements holds.

- There exists $i \in\{1,2\}$ such that $A \prec_{M} M_{i}$.

- We have that $A$ is amenable relative to $B$ inside $M$.

Proof. Assume that $A \nprec_{M} M_{1}$ and $A \nprec_{M} M_{2}$. Denote by $z$ the maximal projection in the center of $A^{\prime} \cap p M p$ such that $A z$ is amenable relative to $B$ inside $M$. If $z=p$, then the theorem is proven. If $z<p$, we replace $p$ by $p-z$ and we replace $A$ by $A(p-z)$. So, $A \nprec_{M} M_{i}$ for all $i \in\{1,2\}$ and for all nonzero projections $q \in \mathcal{Z}\left(A^{\prime} \cap p M p\right)$, we have that $A q$ is not amenable relative to $B$. We refer to this last property by saying that "no corner of $A$ is amenable relative to $B$ inside $M$." We will derive a contradiction.

Exactly as in the proof of [Io12, Theorem 5.1], we define the index set $I$ consisting of all quadruplets $i=(X, Y, \delta, t)$ where $X \subset \widetilde{M}$ and $Y \subset \mathcal{U}(A)$ are finite subsets, $\delta \in(0,1)$ and $t \in(0,1)$. We turn $I$ into a directed set by putting $(X, Y, \delta, t) \leq\left(X^{\prime}, Y^{\prime}, \delta^{\prime}, t^{\prime}\right)$ if and only if $X \subset X^{\prime}, Y \subset Y^{\prime}, \delta^{\prime} \leq \delta$ and $t^{\prime} \leq t$. Since $\theta_{t}(A)$ is amenable relative to $N$ inside $\widetilde{M}$ for all 
$t \in(0,1)$, we can choose for every $i=(X, Y, \delta, t)$ in $I$, a vector $\xi_{i} \in L^{2}\left(\left\langle\widetilde{M}, e_{N}\right\rangle\right)$ such that $\left\|\xi_{i}\right\|_{2} \leq 1$ and

$$
\begin{aligned}
\left|\left\langle x \xi_{i}, \xi_{i}\right\rangle-\tau(x)\right| \leq \delta & \text { whenever } x \in X \quad \text { or } x=\left(\theta_{t}(y)-y\right)^{*}\left(\theta_{t}(y)-y\right) \text { with } y \in Y, \\
\left\|\theta_{t}(y) \xi_{i}-\xi_{i} \theta_{t}(y)\right\|_{2} \leq \delta & \text { whenever } y \in Y .
\end{aligned}
$$

It follows that for all $x \in \widetilde{M}$, we have that $\lim _{i \in I}\left\langle x \xi_{i}, \xi_{i}\right\rangle=\tau(x)$. Since for all $y \in \mathcal{U}(A)$, we have that $\lim _{t \rightarrow 0}\left\|\theta_{t}(y)-y\right\|_{2}=0$, it follows that for all $y \in \mathcal{U}(A)$, we have that $\lim _{i \in I}\left\|y \xi_{i}-\xi_{i} y\right\|_{2}=0$.

Denote by $\mathcal{K}$ the closed linear span of $\left\{x u_{g} e_{N} u_{g}^{*} \mid x \in M, g \in G\right\}$ inside $L^{2}\left(\left\langle\widetilde{M}, e_{N}\right\rangle\right)$. Since $u_{g}^{*} M u_{g} \subset N$, we have that $u_{g} e_{N} u_{g}^{*}$ commutes with $M$ for all $g \in G$. Therefore, $\mathcal{K}$ is an $M-M$ bimodule. Denote by $e$ the orthogonal projection onto $\mathcal{K}$. The net of vectors $\xi_{i}^{\prime}=p(1-e)\left(\xi_{i}\right)$ satisfies $\lim \sup _{i \in I}\left\|x \xi_{i}^{\prime}\right\|_{2} \leq\|x\|_{2}$ for all $x \in p M p$ and $\lim _{i \in I}\left\|a \xi_{i}^{\prime}-\xi_{i}^{\prime} a\right\|_{2}=0$ for all $a \in A$. By Io12, Lemma 4.2], the $M$ - $M$-bimodule $L^{2}\left(\left\langle\widetilde{M}, e_{N}\right\rangle\right) \ominus \mathcal{K}$ is isomorphic with $L^{2}(M) \otimes_{B} \mathcal{L}$ for some $B$ - $M$-bimodule $\mathcal{L}$. Since no corner of $A$ is amenable relative to $B$ inside $M$, it follows from Lemma 2.1 that $\lim _{i \in I}\left\|\xi_{i}^{\prime}\right\|_{2}=0$. So,

$$
\lim _{i \in I}\left\|p \xi_{i}-e\left(p \xi_{i}\right)\right\|_{2}=0
$$

Define the isometry

$$
U: L^{2}(M) \otimes \ell^{2}(G) \rightarrow L^{2}\left(\left\langle\widetilde{M}, e_{N}\right\rangle\right): U\left(x \otimes \delta_{g}\right)=x u_{g} e_{N} u_{g}^{*}
$$

Note that $U U^{*}=e$ and

$$
U((x \otimes 1) \eta(y \otimes 1))=x U(\eta) y \quad \text { for all } x, y \in M, \eta \in L^{2}(M) \otimes \ell^{2}(G) .
$$

We define the net of vectors $\left(\zeta_{i}\right)_{i \in I}$ in $p L^{2}(M) \otimes \ell^{2}(G)$ given by $\zeta_{i}=U^{*}\left(p \xi_{i}\right)$. Note that $\left\|\zeta_{i}\right\|_{2} \leq 1$. The properties of the net $\left(\xi_{i}\right)_{i \in I}$ imply that

$$
\begin{aligned}
\lim _{i \in I}\left\|p \xi_{i}-U\left(\zeta_{i}\right)\right\|_{2} & =0, & & \\
\lim _{i \in I}\left\langle(x \otimes 1) \zeta_{i}, \zeta_{i}\right\rangle & =\tau(p x p) & & \text { for all } x \in M, \\
\lim _{i \in I}\left\|(a \otimes 1) \zeta_{i}-\zeta_{i}(a \otimes 1)\right\|_{2} & =0 & & \text { for all } a \in \mathcal{U}(A) .
\end{aligned}
$$

We view $p L^{2}(M) \otimes \ell^{2}(G)$ as a closed subspace of $L^{2}(\widetilde{M}) \otimes \ell^{2}(G)$. As such, the following claim makes sense.

Claim. For every $\varepsilon>0$, there exists an $s_{0} \in(0,1)$ and an $i_{0} \in I$ such that

$$
\left\|\zeta_{i}-\left(\theta_{s} \otimes \mathrm{id}\right)\left(\zeta_{i}\right)\right\|_{2}<\varepsilon \quad \text { for all } s \in\left[0, s_{0}\right] \text { and all } i \geq i_{0} .
$$

Proof of the claim. Assume the contrary. Using (3.2), we then find an $\varepsilon>0$ such that for every $s \in(0,1)$, we have

$$
\limsup _{i \in I}\left\|\left(\theta_{s} \otimes \mathrm{id}\right)\left(\zeta_{i}\right)-\left(E_{M} \circ \theta_{s} \otimes \mathrm{id}\right)\left(\zeta_{i}\right)\right\|_{2} \geq \varepsilon .
$$

Since for every $a \in \mathcal{U}(A)$, we have $\lim _{s \rightarrow 0}\left\|\theta_{s}(a)-a\right\|_{2}=0$, we can choose a subnet $\left(\mu_{k}\right)$ of the net of vectors

$$
\left((p \otimes 1)\left(\left(\mathrm{id}-E_{M}\right) \circ \theta_{s} \otimes \mathrm{id}\right)\left(\zeta_{i}\right)\right)_{(i, s) \in I \times(0,1)}
$$


with the properties that $\lim \sup _{k}\left\|(x \otimes 1) \mu_{k}\right\|_{2} \leq\|x\|_{2}$ for all $x \in p M p, \liminf _{k}\left\|\mu_{k}\right\|_{2} \geq \varepsilon$ and $\lim _{k}\left\|(a \otimes 1) \mu_{k}-\mu_{k}(a \otimes 1)\right\|_{2}=0$ for all $a \in \mathcal{U}(A)$. The $M$ - $M$-bimodule $L^{2}(\widetilde{M} \ominus M) \otimes$ $\ell^{2}(G)$ is isomorphic with $L^{2}(M) \otimes_{B} \mathcal{L}$ for some $B$-M-bimodule $\mathcal{L}$. By Lemma 2.1, we reach a contradiction with the assumption that no corner of $A$ is amenable relative to $B$ inside $M$. This proves the claim.

Put $\varepsilon=\tau(p) / 14$. Fix $i_{0} \in I$ and $s_{0} \in(0,1)$ such that for all $i \geq i_{0}$ and all $s \in\left[0, s_{0}\right]$, we have that

$$
\left\|p \xi_{i}-U\left(\zeta_{i}\right)\right\|_{2}<\varepsilon \quad \text { and }\left\|\zeta_{i}-\left(\theta_{s} \otimes \mathrm{id}\right)\left(\zeta_{i}\right)\right\|_{2}<\varepsilon .
$$

Write $i_{0}=\left(X_{0}, Y_{0}, \delta_{0}, t_{0}\right)$. Enlarging $i_{0}$ if necessary, we may assume that $p \in X_{0}$, that $p \in Y_{0}$ (note that $p$ is the unit element of $\mathcal{U}(A)$ ), that $\delta_{0}<\varepsilon^{2} / 2$, that $t_{0} \leq s_{0}$ and that $\left\|\theta_{t_{0}}(p)-p\right\|_{2}<$ $\varepsilon / 2$.

Denote by $J$ the index set consisting of all triplets $j=(X, Y, \delta)$, where $X \subset p M p$ and $Y \subset \mathcal{U}(A)$ are finite subsets and $\delta \in\left(0, \delta_{0}\right)$. We turn $J$ into a directed set in a similar way as $I$ above. For every $j=(X, Y, \delta)$, we put

$$
\eta_{j}=\zeta_{\left(X_{0} \cup X, Y_{0} \cup Y, \delta, t_{0}\right)} .
$$

Note that we use here the fixed index $t_{0}$. In particular, $\left(\eta_{j}\right)_{j \in J}$ is not a subnet of $\left(\zeta_{i}\right)_{i \in I}$. Also note that $\left\|\eta_{j}\right\|_{2} \leq 1$. We claim that the net $\left(\eta_{j}\right)_{j \in J}$ of vectors in $p L^{2}(M) \otimes \ell^{2}(G)$ has the following properties.

$$
\begin{array}{ll}
\limsup _{j \in J}\left\|(x \otimes 1) \eta_{j}\right\|_{2} \leq\|x\|_{2} & \text { for all } x \in M, \\
\liminf _{j \in J}\left|\left\langle\theta_{t_{0}}(a) U\left(\eta_{j}\right), U\left(\eta_{j}\right) \theta_{t_{0}}(a)\right\rangle\right| \geq \tau(p)-6 \varepsilon & \text { for all } a \in \mathcal{U}(A), \\
\left\|\eta_{j}-\left(\theta_{s} \otimes \mathrm{id}\right)\left(\eta_{j}\right)\right\|_{2} \leq \varepsilon & \text { for all } s \in\left[0, t_{0}\right] \text { and } j \in J .
\end{array}
$$

To prove (3.3), fix $x \in M$ and fix $j=(X, Y, \delta)$ with $p x^{*} x p \in X$. It suffices to prove that

$$
\left\|(x \otimes 1) \eta_{j}\right\|_{2}^{2} \leq\|x\|_{2}^{2}+\delta .
$$

Put $i=\left(X_{0} \cup X, Y_{0} \cup Y, \delta, t_{0}\right)$. We get that

$$
\left\|(x \otimes 1) \eta_{j}\right\|_{2}=\left\|(x \otimes 1) \zeta_{i}\right\|_{2}=\left\|x U\left(\zeta_{i}\right)\right\|_{2}=\left\|x e\left(p \xi_{i}\right)\right\|_{2}=\left\|e\left(x p \xi_{i}\right)\right\|_{2} \leq\left\|x p \xi_{i}\right\|_{2} .
$$

But also

$$
\left\|x p \xi_{i}\right\|_{2}^{2}=\left\langle p x^{*} x p \xi_{i}, \xi_{i}\right\rangle \leq \tau\left(p x^{*} x p\right)+\delta \leq\|x\|_{2}^{2}+\delta
$$

because $p x^{*} x p \in X \subset X_{0} \cup X$. So 3.6 follows and (3.3) is proven.

To prove (3.4), fix $a \in \mathcal{U}(A)$ and fix $j=(X, Y, \delta)$ with $a \in Y$. It suffices to prove that

$$
\left|\left\langle\theta_{t_{0}}(a) U\left(\eta_{j}\right), U\left(\eta_{j}\right) \theta_{t_{0}}(a)\right\rangle\right| \geq \tau(p)-6 \varepsilon-2 \delta .
$$

Put $i=\left(X_{0} \cup X, Y_{0} \cup Y, \delta, t_{0}\right)$. Since $p \in Y_{0} \subset Y_{0} \cup Y$, we have

$$
\left\|\theta_{t_{0}}(p) \xi_{i}-p \xi_{i}\right\|_{2}^{2} \leq\left\|\theta_{t_{0}}(p)-p\right\|_{2}^{2}+\delta \leq \frac{\varepsilon^{2}}{2}+\delta_{0} \leq \varepsilon^{2} .
$$

So $\left\|\theta_{t_{0}}(p) \xi_{i}-p \xi_{i}\right\|_{2} \leq \varepsilon$. Since $\left\|p \xi_{i}-U\left(\zeta_{i}\right)\right\|_{2} \leq \varepsilon$, we get that

$$
\left\|\theta_{t_{0}}(p) \xi_{i}-U\left(\eta_{j}\right)\right\|_{2} \leq 2 \varepsilon \text {. }
$$


Since $p \in Y_{0} \subset Y_{0} \cup Y$, we also have that $\left\|\theta_{t_{0}}(p) \xi_{i}-\xi_{i} \theta_{t_{0}}(p)\right\|_{2} \leq \delta \leq \delta_{0} \leq \varepsilon$. In combination with the previous inequality, this gives

$$
\left\|\xi_{i} \theta_{t_{0}}(p)-U\left(\eta_{j}\right)\right\|_{2} \leq 3 \varepsilon \text {. }
$$

In the following computation, we write $y \approx_{\varepsilon} z$ when $y, z \in \mathbb{C}$ with $|y-z| \leq \varepsilon$. We also use throughout that $\left\|\zeta_{i}\right\|_{2} \leq 1$ and $\left\|\eta_{j}\right\|_{2} \leq 1$ for all $i \in I$ and $j \in J$. So,

$$
\begin{aligned}
\left\langle\theta_{t_{0}}(a)\right. & \left.U\left(\eta_{j}\right), U\left(\eta_{j}\right) \theta_{t_{0}}(a)\right\rangle & & \\
& \approx_{2 \varepsilon}\left\langle\theta_{t_{0}}(a) \xi_{i}, U\left(\eta_{j}\right) \theta_{t_{0}}(a)\right\rangle & & \text { because }\left\|U\left(\eta_{j}\right)-\theta_{t_{0}}(p) \xi_{i}\right\|_{2} \leq 2 \varepsilon, \\
& \approx_{3 \varepsilon}\left\langle\theta_{t_{0}}(a) \xi_{i}, \xi_{i} \theta_{t_{0}}(a)\right\rangle & & \text { because }\left\|U\left(\eta_{j}\right)-\xi_{i} \theta_{t_{0}}(p)\right\|_{2} \leq 3 \varepsilon, \\
& \approx_{\delta}\left\langle\theta_{t_{0}}(a) \xi_{i}, \theta_{t_{0}}(a) \xi_{i}\right\rangle & & \text { because }\left\|\xi_{i} \theta_{t_{0}}(a)-\theta_{t_{0}}(a) \xi_{i}\right\|_{2} \leq \delta \text { since } a \in Y, \\
& =\left\langle\theta_{t_{0}}(p) \xi_{i}, \xi_{i}\right\rangle & & \\
& \approx_{\varepsilon}\left\langle p \xi_{i}, \xi_{i}\right\rangle & & \text { because }\left\|\theta_{t_{0}}(p) \xi_{i}-p \xi_{i}\right\|_{2} \leq \varepsilon, \\
& \approx_{\delta} \tau(p) & & \text { because } p \in X_{0} \subset X_{0} \cup X .
\end{aligned}
$$

From this computation, (3.7) follows immediately. So also (3.4) is proven.

Finally (3.5) follows because $\left\|\zeta_{i}-\left(\theta_{s} \otimes \mathrm{id}\right)\left(\zeta_{i}\right)\right\|_{2} \leq \varepsilon$ for all $s \in\left[0, t_{0}\right]$ and all $i \geq i_{0}$.

Denote $\eta_{j}=\sum_{g \in G} \eta_{j, g} \otimes \delta_{g}$, where $\eta_{j, g} \in L^{2}(M)$ and where $\left(\delta_{g}\right)_{g \in G}$ is the canonical orthonormal basis of $\ell^{2}(G)$. Recall that for every $x \in L^{2}(\widetilde{M})$ and $h \in G$, we denote $(x)_{h}=E_{N}\left(x u_{h}^{*}\right)$.

For every $a \in \mathcal{U}(A)$, we have that

$$
\sum_{g, h \in G}\left\|\left(\theta_{t_{0}}\left(a \eta_{j, g}\right)\right)_{h}\right\|_{2}^{2}=\sum_{g \in G}\left\|\theta_{t_{0}}\left(a \eta_{j, g}\right)\right\|_{2}^{2}=\sum_{g \in G}\left\|a \eta_{j, g}\right\|_{2}^{2}=\left\|(a \otimes 1) \eta_{j}\right\|_{2}^{2} \leq 1 .
$$

Because the subspaces $\left(L^{2}(N) u_{h g} e_{N} u_{g}^{*}\right)_{h, g \in G}$ of $L^{2}\left(\left\langle\widetilde{M}, e_{N}\right\rangle\right)$ are orthogonal, the formula

$$
\xi(a, j)=\sum_{g, h \in G}\left(\theta_{t_{0}}\left(a \eta_{j, g}\right)\right)_{h} u_{h g} e_{N} u_{g}^{*}
$$

provides a well defined vector in $L^{2}\left(\left\langle\widetilde{M}, e_{N}\right\rangle\right)$ with $\|\xi(a, j)\|_{2} \leq 1$. We claim that for every $a \in \mathcal{U}(A)$ and all $j \in J$, we have

$$
\left\|\theta_{t_{0}}(a) U\left(\eta_{j}\right)-\xi(a, j)\right\|_{2} \leq \varepsilon .
$$

To prove $(3.8)$, first note that

$$
\theta_{t_{0}}(a) U\left(\eta_{j}\right)=\sum_{g \in G} \theta_{t_{0}}(a) \eta_{j, g} u_{g} e_{N} u_{g}^{*}=\sum_{g, h \in G}\left(\theta_{t_{0}}(a) \eta_{j, g}\right)_{h} u_{h g} e_{N} u_{g}^{*} .
$$

It then follows that

$$
\begin{aligned}
\left\|\theta_{t_{0}}(a) U\left(\eta_{j}\right)-\xi(a, j)\right\|_{2}^{2} & =\sum_{g, h \in G}\left\|\left(\theta_{t_{0}}(a) \eta_{j, g}\right)_{h}-\left(\theta_{t_{0}}\left(a \eta_{j, g}\right)\right)_{h}\right\|_{2}^{2} \\
& =\sum_{g \in G}\left\|\theta_{t_{0}}(a) \eta_{j, g}-\theta_{t_{0}}\left(a \eta_{j, g}\right)\right\|_{2}^{2} \\
& =\sum_{g \in G}\left\|\eta_{j, g}-\theta_{t_{0}}\left(\eta_{j, g}\right)\right\|_{2}^{2} \\
& =\left\|\eta_{j}-\left(\theta_{t_{0}} \otimes \mathrm{id}\right)\left(\eta_{j}\right)\right\|_{2}^{2} \leq \varepsilon^{2}
\end{aligned}
$$


So $(3.8)$ is proven.

We similarly define the vectors $\xi^{\prime}(a, j) \in L^{2}\left(\left\langle\widetilde{M}, e_{N}\right\rangle\right)$ by the formula

$$
\xi^{\prime}(a, j)=\sum_{g, h \in G}\left(\theta_{t_{0}}\left(\eta_{j, g} a\right)\right)_{h} u_{g} e_{N} u_{g}^{*} u_{h}=\sum_{g, h \in G}\left(\theta_{t_{0}}\left(\eta_{j, h g} a\right)\right)_{h} u_{h g} e_{N} u_{g}^{*}
$$

and get that $\left\|\xi^{\prime}(a, j)\right\|_{2} \leq 1$ and

$$
\left\|U\left(\eta_{j}\right) \theta_{t_{0}}(a)-\xi^{\prime}(a, j)\right\|_{2} \leq \varepsilon
$$

for all $a \in \mathcal{U}(A)$ and all $j \in J$.

Combining (3.7), (3.8) and $(3.9)$, we find that for all $a \in \mathcal{U}(A)$,

$$
\limsup _{j \in J}\left|\left\langle\xi(a, j), \xi^{\prime}(a, j)\right\rangle\right| \geq \tau(p)-8 \varepsilon .
$$

We now apply Lemma 3.2 and the notation introduced before the formulation of that lemma. For every $a \in \mathcal{U}(A)$ and $j \in J$, we have that

$$
\begin{aligned}
\left\langle\xi(a, j), \xi^{\prime}(a, j)\right\rangle & =\sum_{g, h \in G}\left\langle\left(\theta_{t_{0}}\left(a \eta_{j, g}\right)\right)_{h},\left(\theta_{t_{0}}\left(\eta_{j, h g} a\right)\right)_{h}\right\rangle \\
& =\sum_{g, h \in G} \sum_{\mathcal{I} \in \mathcal{S}}\left\langle P_{\mathcal{I}}\left(a \eta_{j, g}\right), \eta_{j, h g} a\right\rangle \mu_{\mathcal{I}, t_{0}}(h) \\
& =\left\langle Q_{t_{0}}\left((a \otimes 1) \eta_{j}\right), \eta_{j}(a \otimes 1)\right\rangle,
\end{aligned}
$$

where $Q_{t_{0}} \in B\left(L^{2}(M) \otimes \ell^{2}(G)\right)$ is defined by

$$
Q_{t_{0}}=\sum_{\mathcal{I} \in \mathcal{S}} P_{\mathcal{I}} \otimes T_{\mathcal{I}, t_{0}}
$$

So we get that for all $a \in \mathcal{U}(A)$,

$$
\limsup _{j \in J}\left|\left\langle Q_{t_{0}}\left((a \otimes 1) \eta_{j}\right), \eta_{j}(a \otimes 1)\right\rangle\right| \geq \tau(p)-8 \varepsilon .
$$

Fix a large enough integer $n_{0}$ such that $c_{t_{0}}^{n_{0}-1} \leq \varepsilon$. So, $\left\|T_{\mathcal{I}, t_{0}}\right\| \leq \varepsilon$ whenever $|\mathcal{I}| \geq n_{0}$. Denote by

$$
P_{0}=\sum_{\mathcal{I} \in \mathcal{S},|\mathcal{I}|<n_{0}} P_{\mathcal{I}}
$$

the projection onto the closed linear span of "all words of length $<n_{0}$ ".

We claim that there exists a unitary $a \in \mathcal{U}(A)$ such that

$$
\limsup _{j \in J}\left\|\left(P_{0} \otimes 1\right)\left((a \otimes 1) \eta_{j}\right)\right\|_{2} \leq 4 \varepsilon .
$$

To prove this claim, we first use (3.1) to fix $0<s \leq t_{0}$ close enough to zero such that

$$
\left\|\left(P_{0} \otimes 1\right)(\eta)\right\|_{2} \leq 2\left\|\left(E_{M} \otimes \mathrm{id}\right)\left(\left(\theta_{s} \otimes \mathrm{id}\right)(\eta)\right)\right\|_{2} \quad \text { for all } \eta \in L^{2}(M) \otimes \ell^{2}(G) .
$$

Since $A \nprec M_{1}$ and $A \nprec M_{2}$, it follows from Theorem 3.1 that we can choose $a \in \mathcal{U}(A)$ such that $\left\|E_{M}\left(\theta_{s}(a)\right)\right\|_{2} \leq \varepsilon$. We prove that this unitary $a \in \mathcal{U}(A)$ satisfies (3.11). 
From (3.5), we know that $\| \eta_{j}-\left(\theta_{s} \otimes\right.$ id $)\left(\eta_{j}\right) \|_{2} \leq \varepsilon$ for all $j \in J$. It follows that

$$
\left\|\left(\theta_{s} \otimes \mathrm{id}\right)\left((a \otimes 1) \eta_{j}\right)-\left(\theta_{s}(a) \otimes 1\right) \eta_{j}\right\|_{2} \leq \varepsilon \quad \text { for all } j \in J .
$$

So for all $j \in J$, we get that

$$
\begin{aligned}
\left\|\left(P_{0} \otimes 1\right)\left((a \otimes 1) \eta_{j}\right)\right\|_{2} & \leq 2\left\|\left(E_{M} \otimes \mathrm{id}\right)\left(\left(\theta_{s} \otimes \mathrm{id}\right)\left((a \otimes 1) \eta_{j}\right)\right)\right\|_{2} \\
& \leq 2\left\|\left(E_{M} \otimes \mathrm{id}\right)\left(\left(\theta_{s}(a) \otimes 1\right) \eta_{j}\right)\right\|_{2}+2 \varepsilon \\
& =2\left\|\left(E_{M}\left(\theta_{s}(a)\right) \otimes 1\right) \eta_{j}\right\|_{2}+2 \varepsilon .
\end{aligned}
$$

Using (3.3), we get that

$$
\limsup _{j \in J}\left\|\left(P_{0} \otimes 1\right)\left((a \otimes 1) \eta_{j}\right)\right\|_{2} \leq 2\left\|E_{M}\left(\theta_{s}(a)\right)\right\|_{2}+2 \varepsilon \leq 4 \varepsilon .
$$

So the claim in (3.11) is proven and we fix the unitary $a \in \mathcal{U}(A)$ satisfying (3.11).

We now deduce that

$$
\limsup _{j \in J}\left\|Q_{t_{0}}\left((a \otimes 1) \eta_{j}\right)\right\|_{2} \leq 5 \varepsilon
$$

Indeed, since $\left\|T_{\mathcal{I}, t_{0}}\right\| \leq 1$ for all $\mathcal{I} \in \mathcal{S}$ and $\left\|T_{\mathcal{I}, t_{0}}\right\| \leq \varepsilon$ for all $\mathcal{I} \in \mathcal{S}$ with $|\mathcal{I}| \geq n_{0}$, we get that

$$
\begin{aligned}
\left\|Q_{t_{0}}\left((a \otimes 1) \eta_{j}\right)\right\|_{2}^{2} & =\sum_{\mathcal{I} \in \mathcal{S}}\left\|\left(P_{\mathcal{I}} \otimes T_{\mathcal{I}, t_{0}}\right)\left((a \otimes 1) \eta_{j}\right)\right\|_{2}^{2} \\
& \leq \sum_{\mathcal{I} \in \mathcal{S},|\mathcal{I}|<n_{0}}\left\|\left(P_{\mathcal{I}} \otimes 1\right)\left((a \otimes 1) \eta_{j}\right)\right\|_{2}^{2}+\varepsilon^{2} \sum_{\mathcal{I} \in \mathcal{S},|\mathcal{I}| \geq n_{0}}\left\|\left(P_{\mathcal{I}} \otimes 1\right)\left((a \otimes 1) \eta_{j}\right)\right\|_{2}^{2} \\
& \leq\left\|\left(P_{0} \otimes 1\right)\left((a \otimes 1) \eta_{j}\right)\right\|_{2}^{2}+\varepsilon^{2}\left\|(a \otimes 1) \eta_{j}\right\|_{2}^{2} .
\end{aligned}
$$

Taking the limsup over $j \in J$ and using (3.11) and (3.3), we arrive at

$$
\limsup _{j \in J}\left\|Q_{t_{0}}\left((a \otimes 1) \eta_{j}\right)\right\|_{2}^{2} \leq 17 \varepsilon^{2}
$$

and 3.12 follows. But $(3.12)$ implies that

$$
\limsup _{j \in J}\left|\left\langle Q_{t_{0}}\left((a \otimes 1) \eta_{j}\right), \eta_{j}(a \otimes 1)\right\rangle\right| \leq 5 \varepsilon
$$

Since $\varepsilon=\tau(p) / 14$, we have $5 \varepsilon<\tau(p)-8 \varepsilon$ and so we obtained a contradiction with 3.10 .

\section{Proof of Theorem $\mathrm{A}$ and a version for HNN extensions}

Proof of Theorem $A$. We use the malleable deformation $\theta_{t}$ of $M \subset \widetilde{M}$ as explained in Section 3.1. Write $G=\mathbb{F}_{2}$ and $\widetilde{M}=N \rtimes G$ as in Section 3.2 By assumption, $A$ is amenable relative to one of the $M_{i}$ inside $M$. A fortiori, $A$ is amenable relative to $M_{i}$ inside $\widetilde{M}$. Fix $t \in(0,1)$. Applying $\theta_{t}$, we get that $\theta_{t}(A)$ is amenable relative to $\theta_{t}\left(M_{i}\right)$ inside $\widetilde{M}$. Since $\theta_{t}\left(M_{i}\right)$ is unitarily conjugate to $M_{i}$ and $M_{i} \subset N$, it follows that $\theta_{t}(A)$ is amenable relative to $N$ inside $\widetilde{M}$.

Put $P:=\mathcal{N}_{p M p}(A)^{\prime \prime}$. We apply [PV11, Theorem 1.6 and Remark 6.3] to the crossed product $\widetilde{M}=N \rtimes G$ and the subalgebra $\theta_{t}(A)$ of this crossed product. We conclude that at least one of the following statements holds: $\theta_{t}(A) \prec_{\widetilde{M}} N$ or $\theta_{t}(P)$ is amenable relative to $N$ inside $\widetilde{M}$. Since this holds for every $t \in(0,1)$, we get that at least one of the following is true. 
- There exists a $t \in(0,1)$ such that $\theta_{t}(A) \prec \widetilde{M} N$.

- For every $t \in(0,1)$, we have that $\theta_{t}(P)$ is amenable relative to $N$ inside $\widetilde{M}$.

In the first case, Theorem 3.3 implies that $A \prec_{M} B$ or $P \prec_{M} M_{i}$ for some $i \in\{1,2\}$. In the second case, Theorem 3.4 implies that $P \prec_{M} M_{i}$ for some $i \in\{1,2\}$, or that $P$ is amenable relative to $B$ inside $M$.

By [Ue07, HNN extensions can be viewed as corners of amalgamated free products. Since Theorem $\mathrm{A}$ has no particular assumptions on the inclusions $B \subset M_{i}$, we can immediately deduce the following result.

Theorem 4.1. Let $M=\operatorname{HNN}\left(M_{0}, B, \theta\right)$ be the $H N N$ extension of the tracial von Neumann algebra $\left(M_{0}, \tau\right)$ with von Neumann subalgebra $B \subset M_{0}$ and trace preserving embedding $\theta: B \rightarrow$ $M_{0}$. Let $p \in M$ be a nonzero projection and $A \subset p M p$ a von Neumann subalgebra that is amenable relative to $M_{0}$ inside $M$. Then at least one of the following statements holds.

- $A \prec_{M} B$.

- $\mathcal{N}_{p M p}(A)^{\prime \prime} \prec_{M} M_{0}$.

- We have that $\mathcal{N}_{p M p}(A)^{\prime \prime}$ is amenable relative to $B$ inside $M$.

Proof. By [Ue07, Proposition 3.1], we can view $M=\operatorname{HNN}\left(M_{0}, B, \theta\right)$ as a corner of an amalgamated free product. More precisely, we put $M_{1}=\mathrm{M}_{2}(\mathbb{C}) \otimes M_{0}$ and $M_{2}=\mathrm{M}_{2}(\mathbb{C}) \otimes B$. We consider $B_{0}=B \oplus B$ as a subalgebra of both $M_{1}$ and $M_{2}$, where the embedding $B_{0} \hookrightarrow M_{2}$ is diagonal and the embedding $B_{0} \hookrightarrow M_{1}$ is given by $b \oplus d \mapsto b \oplus \theta(d)$. We denote by $e_{i j}$ the matrix units in $M_{1}$ and by $f_{i j}$ the matrix units in $M_{2}$. The HNN extension $M$ is generated by $M_{0}$ and the stable unitary $u$. There is a unique surjective $*$-isomorphism

$$
\Psi: \operatorname{HNN}\left(M_{0}, B, \theta\right) \rightarrow e_{11}\left(M_{1} *_{B_{0}} M_{2}\right) e_{11}:\left\{\begin{array}{l}
\Psi(x)=e_{11} x \\
\Psi(u)=e_{12} f_{21} .
\end{array} \text { for all } x \in M_{0},\right.
$$

Note that in the amalgamated free product, $e_{11}=f_{11}$ and $e_{22}=f_{22}$. Therefore $e_{12} f_{21}$ is really a unitary.

Denote $\mathcal{M}:=M_{1} *_{B_{0}} M_{2}$. Whenever $Q \subset p M p$ is a von Neumann subalgebra, one checks that

- $Q \prec_{M} B$ iff $\Psi(Q) \prec_{\mathcal{M}} B_{0}$ iff $\Psi(Q) \prec_{\mathcal{M}} M_{2}$.

- $Q \prec_{M} M_{0}$ iff $\Psi(Q) \prec_{M} M_{1}$.

- $Q$ is amenable relative to $B$ inside $M$ iff $\Psi(Q)$ is amenable relative to $B_{0}$ inside $\mathcal{M}$.

So Theorem 4.1 is a direct consequence of Theorem $\mathrm{A}$. 


\section{Cartan-rigidity for amalgamated free product groups and HNN extensions}

Recall from [PV11] that a countable group $\Gamma$ is called $\mathcal{C}$-rigid if for every free ergodic pmp action $\Gamma \curvearrowright(X, \mu)$, we have that $L^{\infty}(X)$ is the unique Cartan subalgebra of $L^{\infty}(X) \rtimes \Gamma$ up to unitary conjugacy. Observe that by [Po01, Theorem A.1], $\mathcal{C}$-rigidity is an immediate consequence of the following stronger property $(*)$ :

For every trace preserving action $\Gamma \curvearrowright(B, \tau)$, projection $p \in M=B \rtimes \Gamma$ and amenable von Neumann subalgebra $A \subset p M p$ with $\mathcal{N}_{p M p}(A)^{\prime \prime}=p M p$, we have that $A \prec B$.

As was shown in the proof of [PV12, Theorem 1.1], a direct product $\Gamma_{1} \times \cdots \times \Gamma_{n}$ of finitely many groups $\Gamma_{i}$ with property $(*)$, is $\mathcal{C}$-rigid.

$\operatorname{Property}(*)$ was shown to hold, among other groups, for all weakly amenable $\Gamma$ with $\beta_{1}^{(2)}(\Gamma)>0$ in [PV11, Theorem 7.1] and for all nonelementary hyperbolic $\Gamma$ in [PV12, Theorem 1.4]. In [Io12, Theorem 7.1], property $(*)$ was proven for a large class of amalgamated free products and in [DI12, Proof of Theorem 8.1], for a large class of HNN extensions. For completeness, we show how to deduce these last two results from Theorem A, resp. Theorem 4.1.

Theorem 5.1. The following groups satisfy property $(*)$ and, in particular, are $\mathcal{C}$-rigid.

1. ([Io12, Theorem 7.1]) Amalgamated free products $\Gamma=\Gamma_{1} *_{\Sigma} \Gamma_{2}$ with $\left[\Gamma_{1}: \Sigma\right] \geq 3$ and $\left[\Gamma_{2}: \Sigma\right] \geq 2$ that admit elements $g_{1}, \ldots, g_{n} \in \Gamma$ with $\left|\bigcap_{k=1}^{n} g_{k} \Sigma g_{k}^{-1}\right|<\infty$.

2. ([DI12, Proof of Theorem 8.1]) HNN extensions $\Gamma=\operatorname{HNN}\left(\Gamma_{1}, \Sigma, \theta\right)$, given by a subgroup $\Sigma<\Gamma_{1}$ and an injective group homomorphism $\theta: \Sigma \rightarrow \Gamma_{1}$, such that $\Sigma \neq \Gamma_{1} \neq \theta(\Sigma)$ and such that there exist $g_{1}, \ldots, g_{n} \in \Gamma$ with $\left|\bigcap_{k=1}^{n} g_{k} \Sigma g_{k}^{-1}\right|<\infty$.

Proof. Let $\Gamma \curvearrowright(B, \tau)$ be a trace preserving action and put $M=B \rtimes \Gamma$. Let $p \in M$ be a projection and $A \subset p M p$ an amenable von Neumann subalgebra with $\mathcal{N}_{p M p}(A)^{\prime \prime}=p M p$. In the first case, $M$ is the amalgamated free product of $B \rtimes \Gamma_{1}$ and $B \rtimes \Gamma_{2}$ over $B \rtimes \Sigma$. In the second case, $M$ is the HNN extension of $B \rtimes \Gamma_{1}$ over $B \rtimes \Sigma$. In both cases, $\Gamma_{i}<\Gamma$ has infinite index and $\Sigma<\Gamma$ is not co-amenable (see e.g. the final paragraphs of the proof of [Io12, Theorem 7.1] and [DI12, Lemma 7.2]). So it follows from Theorem A and Theorem 4.1 that $A \prec B \rtimes \Sigma$.

Define the projection $z(\Sigma) \in M \cap(B \rtimes \Sigma)^{\prime}$ as in [HPV10, Section 4]. Since $A \prec B \rtimes \Sigma$, we have that $z(\Sigma) \neq 0$. From [HPV10, Proposition 8], we know that $z(\Sigma)$ belongs to the center of $M$. Take $g_{1}, \ldots, g_{n} \in \Gamma$ such that $\Sigma_{0}=\bigcap_{k=1}^{n} g_{k} \Sigma g_{k}^{-1}$ is a finite group. We have $z\left(g_{k} \Sigma g_{k}^{-1}\right)=u_{g_{k}} z(\Sigma) u_{g_{k}}^{*}=z(\Sigma)$, because $z(\Sigma)$ belongs to the center of $M$. It then follows from [HPV10, Proposition 6] that

$$
z\left(\Sigma_{0}\right)=z\left(g_{1} \Sigma g_{1}^{-1}\right) \cdots z\left(g_{n} \Sigma g_{n}^{-1}\right)=z(\Sigma) \neq 0 .
$$

So $A \prec B \rtimes \Sigma_{0}$. Since $\Sigma_{0}$ is finite, we conclude that $A \prec B$.

\section{Proof of Corollary B}

Proof of Corollary B. Write $M_{i}=L\left(\mathcal{R}_{i}\right)$ and $B=L^{\infty}(X)$. Note that $L(\mathcal{R})=M_{1} *_{B} M_{2}$. Corollary $\mathrm{B}$ is a direct consequence of Theorem $\mathrm{A}$, provided that we prove the following two statements. 
1. $M \nprec_{M} M_{i}$.

2. $M$ is not amenable relative to $B$, i.e. $M$ is not amenable itself.

Since $\left|\mathcal{R}_{1} \cdot x\right| \geq 3$ for a.e. $x \in X$ and using e.g. [IKT08, Lemma 2.6], we can take unitaries $u, v \in \mathcal{U}\left(M_{1}\right)$ such that $E_{B}(u)=E_{B}(v)=E_{B}\left(u^{*} v\right)=0$. We similarly find a unitary $w \in \mathcal{U}\left(M_{2}\right)$ with $E_{B}(w)=0$.

Proof of 1 . Define the sequence of unitaries $w_{n} \in \mathcal{U}(M)$ given by $w_{n}=(u w)^{n}$. Denote by $X_{m} \subset M$ the linear span of all products of at most $m$ elements from $M_{1} \ominus B$ and $M_{2} \ominus B$. Whenever $2 n>2 m+1$ and $x, y \in X_{m}$, a direct computation yields that $E_{M_{i}}\left(x w_{n} y\right)=0$. So it follows that $\lim _{n}\left\|E_{M_{i}}\left(x w_{n} y\right)\right\|_{2}=0$ for all $x, y \in M$ and 1 follows.

Proof of 2. Assume that $M$ is amenable and take an $M$-central state $\Omega$ on $B\left(L^{2}(M)\right)$. Define $K_{1}$ as the closed linear span of $B$ and all products of the form $x_{1} x_{2} \cdots x_{n}$ with $x_{1} \in M_{1} \ominus B$, $x_{2} \in M_{2} \ominus B$, etc. Define $K_{2}$ as the closed linear span of all products of the form $y_{1} y_{2} \cdots y_{n}$ with $y_{1} \in M_{2} \ominus B, y_{2} \in M_{1} \ominus B$, etc. By construction, $L^{2}(M)=K_{1} \oplus K_{2}$. Denote by $e_{i}$ the orthogonal projection of $L^{2}(M)$ onto $K_{i}$. It follows that $u e_{2} u^{*}$ and $v e_{2} v^{*}$ are orthogonal and lie under $e_{1}$. Hence, $2 \Omega\left(e_{2}\right)=\Omega\left(u e_{2} u^{*}\right)+\Omega\left(v e_{2} v^{*}\right) \leq \Omega\left(e_{1}\right)$. On the other hand, $w e_{1} w^{*} \leq e_{2}$, implying that $\Omega\left(e_{1}\right)=\Omega\left(w e_{1} w^{*}\right) \leq \Omega\left(e_{2}\right)$. Altogether it follows that $\Omega\left(e_{1}\right)=\Omega\left(e_{2}\right)=0$. Since $1=e_{1}+e_{2}$ and $\Omega(1)=1$, we reached a contradiction.

\section{Proof of Corollary $\mathrm{C}$}

Proof of Corollary $C$. Let $A \subset M$ be a diffuse amenable von Neumann subalgebra. Denote $P=\mathcal{N}_{M}(A)^{\prime \prime}$ and assume that $P$ is not amenable. Take a nonzero central projection $z \in \mathcal{Z}(P)$ such that $P z$ has no amenable direct summand. Since $P z \subset \mathcal{N}_{z M z}(A z)^{\prime \prime}$, it follows from Theorem $\mathrm{A}$ that one of the following statements holds.

1. $A z \prec_{M} B$.

2. $P z \prec_{M} M_{i}$ for some $i \in\{1,2\}$.

3. $P z$ is amenable relative to $B$ inside $M$.

It suffices to prove that each of the three statements is false.

1. Observe that the inclusion $M_{2} \subset M$ is mixing. To prove this, fix a sequence $b_{n}$ in the unit ball of $M_{2}$ such that $b_{n} \rightarrow 0$ weakly. We must show that $\lim _{n}\left\|E_{M_{2}}\left(x^{*} b_{n} y\right)\right\|_{2}=0$ for all $x, y \in M \ominus M_{2}$. It suffices to prove this when $x=x_{1} x_{2} \cdots x_{n}$ and $y=y_{1} y_{2} \cdots y_{m}$ with $n, m \geq 2$, $x_{1}, y_{1} \in M_{2}, x_{2}, y_{2} \in M_{1} \ominus B, x_{3}, y_{3} \in M_{2} \ominus B$, etc. But then

$$
E_{M_{2}}\left(x^{*} b_{n} y\right)=E_{M_{2}}\left(x_{n}^{*} \cdots x_{3}^{*} E_{B}\left(x_{2}^{*} E_{B}\left(x_{1}^{*} b_{n} y_{1}\right) y_{2}\right) y_{3} \cdots y_{n}\right)
$$

and the conclusion follows because $E_{B}\left(x_{1}^{*} b_{n} y_{1}\right) \rightarrow 0$ weakly and the inclusion $B \subset M_{1}$ is mixing.

Assume that 1 holds. Then certainly $A z \prec_{M} M_{2}$. Since the inclusion $M_{2} \subset M$ is mixing, it follows from [Io12, Lemma 9.4] that $P z \prec_{M} M_{2}$. So statement 2 holds and we proceed to the next point.

2. Assume that 2 holds. We then find in particular a nonzero projection $p \in M_{n}(\mathbb{C}) \otimes M_{i}$ and a normal unital $*$-homomorphism $\varphi: P z \rightarrow p\left(M_{n}(\mathbb{C}) \otimes M_{i}\right) p$. Then $\varphi(A z)$ is a diffuse von Neumann subalgebra of $p\left(M_{n}(\mathbb{C}) \otimes M_{i}\right) p$ whose normalizer contains $\varphi(P z)$. Since $P z$ has no 
amenable direct summand, $\varphi(P z)$ is nonamenable. Hence $p\left(M_{n}(\mathbb{C}) \otimes M_{i}\right) p$ is not strongly solid. Since $M_{i}$ is strongly solid, this contradicts the stability of strong solidity under amplifications as proven in [Ho09, Proposition 5.2].

3. Since $B$ is amenable, 3 implies that $P z$ is amenable, contradicting our assumptions.

\section{8. $\mathrm{W}^{*}$-superrigid actions of type III}

In the same way as [HV12, Theorem A] was deduced from the results in [PV12, we can deduce from Theorem A the following type III uniqueness statement for Cartan subalgebras. Our theorem is a generalization of [BHR12, Theorem D], where the same result was proven under the assumption that $\Sigma$ is a finite group.

Rather than looking for the most general statement possible, we provide a more ad hoc formulation that suffices to prove the $\mathrm{W}^{*}$-superrigidity of the type $\mathrm{III}_{1}$ actions in Proposition $\mathrm{D}$ (see also Remark 8.3 below). Recall that a nonsingular action $\Lambda \curvearrowright(X, \mu)$ is said to be recurrent if there is no Borel subset $\mathcal{U} \subset X$ such that $\mu(\mathcal{U})>0$ and $\mu(g \cdot \mathcal{U} \cap \mathcal{U})=0$ for all $g \in \Lambda-\{e\}$.

Theorem 8.1. Let $\Gamma=\Gamma_{1} *_{\Sigma} \Gamma_{2}$ be an amalgamated free product group and assume that there exist $g_{1}, \ldots, g_{n} \in \Gamma$ such that $\bigcap_{k=1}^{n} g_{k} \Sigma g_{k}^{-1}$ is finite.

Let $\Gamma \curvearrowright(X, \mu)$ be any nonsingular free ergodic action. Assume that each $\Gamma_{i}$ admits a subgroup $\Lambda_{i}$ such that the restricted action $\Lambda_{i} \curvearrowright(X, \mu)$ is recurrent and $\Lambda_{i} \cap \Sigma$ is finite.

Then, $L^{\infty}(X)$ is the unique Cartan subalgebra of $L^{\infty}(X) \rtimes \Gamma$ up to unitary conjugacy.

For Theorem 8.1 to hold, it is essential to impose some recurrence of $\Gamma_{i} \curvearrowright(X, \mu)$ relative to $\Sigma$. Indeed, otherwise the action $\Gamma \curvearrowright(X, \mu)$ could simply be the induction of an action $\Gamma_{i} \curvearrowright(Z, \eta)$ so that $L^{\infty}(X) \rtimes \Gamma \cong B(H) \bar{\otimes}\left(L^{\infty}(Z) \rtimes \Gamma_{i}\right)$ and we cannot expect uniqueness of the Cartan subalgebra.

Before proving Theorem 8.1, we provide a semifinite variant of the machinery developed in [HPV10, Sections 4 and 5]. We start by the following elementary lemma and leave the proof to the reader.

Lemma 8.2. Let $(N, \mathrm{Tr})$ be a von Neumann algebra equipped with a normal semifinite faithful trace. Let $H$ be a right Hilbert $N$-module and $p \in N$ a projection. We consider dimensions using the trace $\operatorname{Tr}$ and its restrictions to subalgebras of $N$ and $p N p$.

1. We have $\operatorname{dim}_{p N p}(H p) \leq \operatorname{dim}_{N}(H)$.

2. Let $K \subset H p$ be a closed $p N p$-submodule. Then $\operatorname{dim}_{N}(\operatorname{closure}(K N))=\operatorname{dim}_{p N p}(K)$.

3. Let $P \subset N$ be a von Neumann subalgebra such that $\operatorname{Tr}_{\mid P}$ is semifinite. Let $K \subset H$ be a closed P-submodule. Then $\operatorname{dim}_{N}(\operatorname{closure}(K N)) \leq \operatorname{dim}_{P}(K)$.

Assume that $\Gamma$ is a countable group and $\Gamma \curvearrowright(B, \operatorname{Tr})$ a trace preserving action on a von Neumann algebra $B$ equipped with a normal semifinite faithful trace Tr. Denote $\mathcal{M}=B \rtimes \Gamma$ and use the canonical trace $\operatorname{Tr}$ on $\mathcal{M}$. Let $p \in \mathcal{M}$ be a projection with $\operatorname{Tr}(p)<\infty$ and $A \subset p \mathcal{M} p$ a von Neumann subalgebra with $\mathcal{N}_{p \mathcal{M} p}(A)^{\prime \prime}=p \mathcal{M} p$. Whenever $\Lambda<\Gamma$ is a subgroup, we consider

$$
\mathcal{E}_{\Lambda}=\left\{H \mid H \text { is an } A-(B \rtimes \Lambda) \text {-subbimodule of } L^{2}(p \mathcal{M}) \text { with } \operatorname{dim}_{B \rtimes \Lambda}(H)<\infty\right\} .
$$


If $H \in \mathcal{E}_{\Lambda}, u \in \mathcal{N}_{p \mathcal{M} p}(A)$ and $v \in \mathcal{U}(B \rtimes \Lambda)$, we have that $u H v$ again belongs to $\mathcal{E}_{\Lambda}$. So the closed linear span of all $H \in \mathcal{E}_{\Lambda}$ is of the form $L^{2}(p \mathcal{M} z(\Lambda))$, where $z(\Lambda)$ is a projection in $\mathcal{M} \cap(B \rtimes \Lambda)^{\prime}$. We make $z(\Lambda)$ uniquely determined by requiring that $z(\Lambda)$ is smaller than or equal to the central support of $p$ in $\mathcal{M}$.

If $\Lambda<\Lambda^{\prime}<\Gamma$ are subgroups, we have $z(\Lambda) \leq z\left(\Lambda^{\prime}\right)$. Indeed, whenever $H \subset L^{2}(p \mathcal{M})$ is an $A$ - $(B \rtimes \Lambda)$-subbimodule with $\operatorname{dim}_{B \rtimes \Lambda}(H)<\infty$, we define $K$ as the closed linear span of $H\left(B \rtimes \Lambda^{\prime}\right)$. By Lemma 8.2 , we get that $\operatorname{dim}_{B \rtimes \Lambda^{\prime}}(K)<\infty$. Since $H \subset K$ and since this works for all choices of $H$, we conclude that $z(\Lambda) \leq z\left(\Lambda^{\prime}\right)$.

The basic construction $\left\langle\mathcal{M}, e_{B \rtimes \Lambda}\right\rangle$ carries a natural semifinite trace $\operatorname{Tr}$ satisfying $\operatorname{Tr}\left(x e_{B \rtimes \Lambda} x^{*}\right)=$ $\operatorname{Tr}\left(x x^{*}\right)$ for all $x \in \mathcal{M}$. The projections $e \in A^{\prime} \cap p\left\langle\mathcal{M}, e_{B \rtimes \Lambda}\right\rangle p$ are precisely the orthogonal projections onto the $A$ - $(B \rtimes \Lambda)$-subbimodules $H \subset L^{2}(p \mathcal{M})$. Moreover under this correspondence, we have $\operatorname{Tr}(e)=\operatorname{dim}_{B \rtimes \Lambda}(H)$. We also have the canonical operator valued weight $\mathcal{T}_{\Lambda}$ from $\left\langle\mathcal{M}, e_{B \rtimes \Lambda}\right\rangle^{+}$to the extended positive part of $\mathcal{M}$ such that $\operatorname{Tr}=\operatorname{Tr} \circ \mathcal{T}_{\Lambda}$. Using the anti-unitary involution $J: L^{2}(\mathcal{M}) \rightarrow L^{2}(\mathcal{M}): J(x)=x^{*}$, we can therefore alternatively define $z(\Lambda)$ as

$$
\begin{aligned}
p J z(\Lambda) J & =\bigvee\left\{e \mid e \in A^{\prime} \cap p\left\langle\mathcal{M}, e_{B \rtimes \Lambda}\right\rangle p \text { is a projection with }\left\|\mathcal{T}_{\Lambda}(e)\right\|<\infty\right\} \\
& =\bigvee\left\{\operatorname{supp}(a) \mid a \in A^{\prime} \cap p\left\langle\mathcal{M}, e_{B \rtimes \Lambda}\right\rangle^{+} p \text { and }\left\|\mathcal{T}_{\Lambda}(a)\right\|<\infty\right\} .
\end{aligned}
$$

If now $\Lambda<\Gamma$ and $\Lambda^{\prime}<\Gamma$ are subgroups, we can literally repeat the proof of HPV10, Proposition $6]$ and conclude that $z(\Lambda)$ and $z\left(\Lambda^{\prime}\right)$ commute with

$$
z\left(\Lambda \cap \Lambda^{\prime}\right)=z(\Lambda) z\left(\Lambda^{\prime}\right) .
$$

We are now ready to prove Theorem 8.1 .

Proof of Theorem 8.1. Denote by $\omega: \Gamma \times X \rightarrow \mathbb{R}$ the logarithm of the Radon-Nikodym cocycle. Put $Y=X \times \mathbb{R}$ and equip $Y$ with the measure $m$ given by $d m=d \mu \times \exp (t) d t$, so that the action $\Gamma \curvearrowright Y$ given by $g \cdot(x, t)=(g \cdot x, \omega(g, x)+t)$ is measure preserving (see [Ma63]). The restricted actions $\Lambda_{i} \curvearrowright(Y, m)$ are still recurrent.

Put $B=L^{\infty}(Y)$ and denote by $\operatorname{Tr}$ the canonical semifinite trace on $\mathcal{M}=B \rtimes \Gamma$, given by the infinite invariant measure $m$. Choose a projection $p \in B$ with $0<\operatorname{Tr}(p)<\infty$. Put $\Sigma_{i}=\Lambda_{i} \cap \Sigma$. Since $\Sigma_{i}$ is a finite group, the von Neumann algebra $p\left(B \rtimes \Sigma_{i}\right) p$ is of type I. Since the action $\Lambda_{i} \curvearrowright(Y, m)$ is recurrent, the von Neumann algebra $p\left(B \rtimes \Lambda_{i}\right) p$ is of type $\mathrm{II}_{1}$. In particular, the inclusion $p\left(B \rtimes \Sigma_{i}\right) p \subset p\left(B \rtimes \Lambda_{i}\right) p$ has no trivial corner in the sense of [HV12, Definition 5.1] and it follows from [HV12, Lemma 5.4] that there exists a unitary $u_{i} \in p\left(B \rtimes \Lambda_{i}\right) p$ such that $E_{p\left(B \rtimes \Sigma_{i}\right) p}\left(u_{i}^{n}\right)=0$ for all $n \in \mathbb{Z}-\{0\}$. Since $\Lambda_{i} \cap \Sigma=\Sigma_{i}$, we have that $E_{p(B \rtimes \Sigma) p}(x)=E_{p\left(B \rtimes \Sigma_{i}\right) p}(x)$ for all $x \in p\left(B \rtimes \Lambda_{i}\right) p$. So, we get that $E_{p(B \rtimes \Sigma) p}\left(u_{i}^{n}\right)=0$ for all $n \in \mathbb{Z}-\{0\}$. We put $v_{i}=u_{i}^{*}$ and have found unitaries $u_{i}, v_{i} \in p\left(B \rtimes \Gamma_{i}\right) p$ satisfying

$$
E_{p(B \rtimes \Sigma) p}\left(u_{i}\right)=E_{p(B \rtimes \Sigma) p}\left(v_{i}\right)=E_{p(B \rtimes \Sigma) p}\left(u_{i}^{*} v_{i}\right)=0 .
$$

Define the normal trace preserving $*$-homomorphism

$$
\Delta: \mathcal{M} \rightarrow \mathcal{M} \bar{\otimes} L(\Gamma): \Delta\left(b u_{g}\right)=b u_{g} \otimes u_{g} \quad \text { for all } b \in B, g \in \Gamma .
$$

We use the unitaries $u_{i}$ satisfying $(8.2)$ to prove the following two easy statements.

Statement 1. For every $i=1,2$, we have that $\Delta(p \mathcal{M} p) \nprec_{p \mathcal{M} p \bar{\otimes} L(\Gamma)} p \mathcal{M} p \bar{\otimes} L\left(\Gamma_{i}\right)$. 
Statement 2. The von Neumann subalgebra $\Delta(p \mathcal{M} p) \subset p \mathcal{M} p \bar{\otimes} L(\Gamma)$ is not amenable relative to $p \mathcal{M} p \bar{\otimes} L(\Sigma)$.

Proof of statement 1. Denote by $|g|$ the length of an element $g \in \Gamma$, i.e. the minimal number of factors that is needed to write $g$ as a product of elements in $\Gamma_{1}, \Gamma_{2}$, with the convention that $|g|=0$ if and only if $g \in \Sigma$. Denote by $Q_{m}$ the orthogonal projection of $L^{2}(p \mathcal{M} p)$ onto the closed linear span of $\left\{p b u_{g} p|b \in B, g \in \Gamma| g \mid, \leq m\right\}$. Denote by $P_{m}$ the orthogonal projection of $\ell^{2}(\Gamma)$ onto the closed linear span of $\left\{u_{g}|g \in \Gamma| g \mid, \leq m\right\}$. A direct computation yields

$$
\left(1 \otimes P_{m}\right)(\Delta(x))=\Delta\left(Q_{m}(x)\right) \text { for all } x \in p \mathcal{M} p .
$$

Define the unitary $w_{n}=\left(u_{1} u_{2}\right)^{n}$. Since $Q_{m}\left(w_{n}\right)=0$ whenever $n>m / 2$, we have that $\left(1 \otimes P_{m}\right)\left(\Delta\left(w_{n}\right)\right)=0$ for all $n>m / 2$. It follows in particular that for all $g, h \in \Gamma$,

$$
E_{p \mathcal{M} p \bar{\otimes} L\left(\Gamma_{i}\right)}\left(\left(1 \otimes u_{g}\right) \Delta\left(w_{n}\right)\left(1 \otimes u_{h}\right)\right)=0 \quad \text { whenever } n>(|g|+|h|+1) / 2 .
$$

So, for every $x, y \in p \mathcal{M} p \bar{\otimes} L(\Gamma)$, we get that $\lim _{n}\left\|E_{p \mathcal{M} p \bar{\otimes} L\left(\Gamma_{i}\right)}\left(x \Delta\left(w_{n}\right) y\right)\right\|_{2}=0$. Hence, $\Delta(p \mathcal{M} p) \nprec p \mathcal{M} p \bar{\otimes} L\left(\Gamma_{i}\right)$ and statement 1 is proven.

Proof of statement 2. Assume that $\Delta(p \mathcal{M} p)$ is amenable relative $p \mathcal{M} p \bar{\otimes} L(\Sigma)$. So we find a positive $\Delta(p \mathcal{M} p)$-central functional $\Omega$ on $\left\langle p \mathcal{M} p \bar{\otimes} L(\Gamma), e_{p \mathcal{M} p \bar{\otimes} L(\Sigma)}\right\rangle$ such that $\Omega(x)=(\operatorname{Tr} \otimes \tau)(x)$ for all $x \in p \mathcal{M} p \bar{\otimes} L(\Gamma)$. Note that we can identify

$$
\left\langle p \mathcal{M} p \bar{\otimes} L(\Gamma), e_{p \mathcal{M} p \bar{\otimes} L(\Sigma)}\right\rangle=p \mathcal{M} p \bar{\otimes}\left\langle L(\Gamma), e_{L(\Sigma)}\right\rangle=(p \otimes 1)\langle\mathcal{M} \bar{\otimes} L(\Gamma), \mathcal{M} \bar{\otimes} L(\Sigma)\rangle(p \otimes 1) .
$$

Since $E_{\mathcal{M} \bar{\otimes} L(\Sigma)} \circ \Delta=\Delta \circ E_{B \rtimes \Sigma}$ and since the closed linear span of $\Delta(\mathcal{M}) L^{2}(\mathcal{M} \bar{\otimes} L(\Sigma))$ equals $L^{2}(\mathcal{M} \bar{\otimes} L(\Gamma))$, there is a unique normal unital $*$-homomorphism

$\Psi:\left\langle\mathcal{M}, e_{B \rtimes \Sigma}\right\rangle \rightarrow\left\langle\mathcal{M} \bar{\otimes} L(\Gamma), e_{\mathcal{M} \bar{\otimes} L(\Sigma)}\right\rangle: \Psi\left(x e_{B \rtimes \Sigma} y\right)=\Delta(x) e_{\mathcal{M} \bar{\otimes} L(\Sigma)} \Delta(y) \quad$ for all $x, y \in \mathcal{M}$.

The composition of $\Omega$ and $\Psi$ yields a $p \mathcal{M} p$-central positive functional $\Omega_{0}$ on $p\left\langle\mathcal{M}, e_{B \rtimes \Sigma}\right\rangle p$ satisfying $\Omega_{0}(p)=\operatorname{Tr}(p)$. Note that we can view $p\left\langle\mathcal{M}, e_{B \rtimes \Sigma}\right\rangle p$ as the commutant of the right action of $B \rtimes \Sigma$ on $p L^{2}(\mathcal{M})$.

Denote by $H_{i} \subset p L^{2}(\mathcal{M})$ the closed linear span of all $p b u_{g}$ with $b \in B$ and $g \in \Gamma$ such that a reduced expression of $\Gamma$ as an alternating product of elements in $\Gamma_{1}-\Sigma$ and $\Gamma_{2}-\Sigma$ starts with a factor in $\Gamma_{i}-\Sigma$. Denote $H_{0}=p L^{2}(B)$. So we have the orthogonal decomposition $p L^{2}(\mathcal{M})=H_{0} \oplus H_{1} \oplus H_{2}$. Denote by $e_{i}: p L^{2}(\mathcal{M}) \rightarrow H_{i}$ the orthogonal projection. Note that $e_{i}$ is a projection in $p\left\langle\mathcal{M}, e_{B \rtimes \Sigma}\right\rangle p$. By $(8.2)$, the projections $u_{2}\left(e_{0}+e_{1}\right) u_{2}^{*}$ and $v_{2}\left(e_{0}+e_{1}\right) v_{2}^{*}$ are orthogonal and lie under $e_{2}$. Since $\Omega_{0}$ is $p \mathcal{M} p$-central, it follows that

$$
2 \Omega_{0}\left(e_{0}+e_{1}\right) \leq \Omega_{0}\left(e_{2}\right)
$$

It similarly follows that $2 \Omega_{0}\left(e_{2}\right) \leq \Omega_{0}\left(e_{1}\right)$. Together, it follows that $\Omega_{0}\left(e_{0}+e_{1}\right)=\Omega_{0}\left(e_{2}\right)=0$. Since $e_{0}+e_{1}+e_{2}=p$, we obtain the contradiction that $\Omega_{0}(p)=0$. So also statement 2 is proven.

Assume now that $L^{\infty}(X) \rtimes \Gamma$ admits a Cartan subalgebra that is not unitarily conjugate to $L^{\infty}(X)$. The first paragraphs of the proof of [HV12, Theorem A] are entirely general and yield an abelian von Neumann subalgebra $A \subset p \mathcal{M} p$ such that $\mathcal{N}_{p \mathcal{M} p}(A)^{\prime \prime}=p \mathcal{M} p$ and $A \nprec B q$ whenever $q \in B$ is a projection with $\operatorname{Tr}(q)<\infty$. So to prove the theorem, we fix an abelian von Neumann subalgebra $A \subset p \mathcal{M} p$ with $\mathcal{N}_{p \mathcal{M} p}(A)^{\prime \prime}=p \mathcal{M} p$. We have to find a projection $q \in B$ with $\operatorname{Tr}(q)<\infty$ and $A \prec B q$. 
Note that $\Delta(A) \subset p \mathcal{M} p \bar{\otimes} L(\Gamma)$ is an abelian, hence amenable, von Neumann subalgebra whose normalizer contains $\Delta(p \mathcal{M} p)$. We view $p \mathcal{M} p \bar{\otimes} L(\Gamma)$ as the amalgamated free product of $p \mathcal{M} p \bar{\otimes} L\left(\Gamma_{1}\right)$ and $p \mathcal{M} p \bar{\otimes} L\left(\Gamma_{2}\right)$ over their common von Neumann subalgebra $p \mathcal{M} p \bar{\otimes} L(\Sigma)$. A combination of Theorem $\mathrm{A}$ and statements 1 and 2 above implies that $\Delta(A) \prec p \mathcal{M} p \bar{\otimes} L(\Sigma)$. So there is no sequence of unitaries $\left(a_{n}\right)$ in $\mathcal{U}(A)$ satisfying $\lim _{n}\left\|E_{p \mathcal{M} p \bar{\otimes} L(\Sigma)}\left(x \Delta\left(a_{n}\right) y\right)\right\|_{2}=0$ for all $x, y \in p \mathcal{M} p \bar{\otimes} L(\Gamma)$. This means that we can find $\varepsilon>0$ and $h_{1}, \ldots, h_{m} \in \Gamma$ such that

$$
\sum_{i, j=1}^{m}\left\|E_{p \mathcal{M} p \bar{\otimes} L(\Sigma)}\left(\left(1 \otimes u_{h_{i}}^{*}\right) \Delta(a)\left(1 \otimes u_{h_{j}}\right)\right)\right\|_{2}^{2} \geq \varepsilon \quad \text { for all } a \in \mathcal{U}(A) .
$$

Define the positive element $T=\sum_{i=1}^{m} p u_{h_{i}} e_{B \rtimes \Sigma} u_{h_{i}}^{*} p$ in $p\left\langle\mathcal{M}, e_{B \rtimes \Sigma}\right\rangle p$. The left hand side of (8.3) equals $\operatorname{Tr}\left(\mathrm{TaTa}^{*}\right)$. Denote by $S$ the element of smallest $\|\cdot\|_{2, \operatorname{Tr}}$-norm in the weakly closed convex hull of $\left\{a T a^{*} \mid a \in \mathcal{U}(A)\right\}$. Then $S$ is a nonzero element of $A^{\prime} \cap p\left\langle\mathcal{M}, e_{B \rtimes \Sigma}\right\rangle p$ and $\operatorname{Tr}(S)<\infty$. Using the notation introduced before this proof, this means that $z(\Sigma) \neq 0$.

Since the action $\Gamma \curvearrowright Y$ is free, we have $\mathcal{M} \cap B^{\prime}=B$. So the projections $z(\Sigma)$ and $z\left(\Gamma_{i}\right)$ belong to $B$ and are, respectively, $\Sigma$ - and $\Gamma_{i}$-invariant. We prove below that $z(\Sigma)$ is a $\Gamma$-invariant projection in $B$. We prove this by showing that $z\left(\Gamma_{1}\right)=z(\Sigma)=z\left(\Gamma_{2}\right)$.

Since $\Sigma<\Gamma_{i}$, we have that $z(\Sigma) \leq z\left(\Gamma_{i}\right)$ for every $i=1,2$. We claim that the equality holds. Assume that $z(\Sigma)<z\left(\Gamma_{1}\right)$. Note that both projections belong to $B$. Choose a nonzero projection $q \in B$ with $\operatorname{Tr}(q)<\infty$ and $q \leq z\left(\Gamma_{1}\right)-z(\Sigma)$. Choose $H \in \mathcal{E}_{\Gamma_{1}}$ such that $H q \neq\{0\}$. By Lemma 8.2 , we have

$$
\operatorname{dim}_{q\left(B \rtimes \Gamma_{1}\right) q}(H q) \leq \operatorname{dim}_{B \rtimes \Gamma_{1}}(H)<\infty .
$$

We conclude that $L^{2}(p \mathcal{M} q)$ admits a nonzero $A-q\left(B \rtimes \Gamma_{1}\right) q$-subbimodule $K$ that is finitely generated as a right Hilbert module. Since $q \perp z(\Sigma)$, we also know that $L^{2}(p \mathcal{M} q)$ does not admit an $A-q(B \rtimes \Sigma) q$-subbimodule that is finitely generated as a right Hilbert module. We then encode $K$ as an integer $n$, a projection $q_{1} \in M_{n}(\mathbb{C}) \otimes q\left(B \rtimes \Gamma_{1}\right) q$, a nonzero partial isometry $V \in p\left(M_{1, n}(\mathbb{C}) \otimes \mathcal{M}\right) q_{1}$ and a normal unital $*$-homomorphism $\varphi: A \rightarrow q_{1}\left(M_{n}(\mathbb{C}) \otimes\left(B \rtimes \Gamma_{1}\right)\right) q_{1}$ such that

$$
a V=V \varphi(a) \text { for all } a \in A \text { and } \varphi(A) \nprec_{M_{n}(\mathbb{C}) \otimes q\left(B \rtimes \Gamma_{1}\right) q} q(B \rtimes \Sigma) q .
$$

Let $u \in \mathcal{N}_{p \mathcal{M} p}(A)$ and write $u a u^{*}=\alpha(a)$ for all $a \in A$. Then $V^{*} u V$ is an element of $q_{1}\left(M_{n}(\mathbb{C}) \otimes\right.$ $\mathcal{M}) q_{1}$ satisfying

$$
V^{*} u V \varphi(a)=\varphi(\alpha(a)) V^{*} u V \text { for all } a \in A .
$$

By (8.4) and [CH08, Theorem 2.4], it follows that $V^{*} u V \in q_{1}\left(M_{n}(\mathbb{C}) \otimes\left(B \rtimes \Gamma_{1}\right)\right) q_{1}$. This holds for all $u \in \mathcal{N}_{p \mathcal{M} p}(A)$. Since the linear span of $\mathcal{N}_{p \mathcal{M} p}(A)$ is strongly dense in $p \mathcal{M} p$, and writing $q_{2}=V^{*} V$, we have found a nonzero projection $q_{2} \in M_{n}(\mathbb{C}) \otimes\left(B \rtimes \Gamma_{1}\right)$ with the property that

$$
q_{2}\left(M_{n}(\mathbb{C}) \otimes \mathcal{M}\right) q_{2}=q_{2}\left(M_{n}(\mathbb{C}) \otimes\left(B \rtimes \Gamma_{1}\right)\right) q_{2} .
$$

In the von Neumann algebra $M_{n}(\mathbb{C}) \otimes\left(B \rtimes \Gamma_{1}\right)$, the projection $q_{2}$ is equivalent with a projection in $D_{n}(\mathbb{C}) \otimes B$, where $D_{n}(\mathbb{C}) \subset M_{n}(\mathbb{C})$ is the diagonal subalgebra. So, we find a nonzero projection $q_{3} \in B$ satisfying $q_{3} \mathcal{M} q_{3}=q_{3}\left(B \rtimes \Gamma_{1}\right) q_{3}$. As in $(8.2)$, there however exists a unitary $v \in q_{3}\left(B \rtimes \Gamma_{2}\right) q_{3}$ with the property that $E_{q_{3}(B \rtimes \Sigma) q_{3}}(v)=0$. It follows that $v$ belongs to $q_{3} \mathcal{M} q_{3}$, but is orthogonal to $q_{3}\left(B \rtimes \Gamma_{1}\right) q_{3}$. We have reached a contradiction and conclude that $z(\Sigma)=z\left(\Gamma_{1}\right)$. By symmetry, we also have that $z(\Sigma)=z\left(\Gamma_{2}\right)$.

Since $z\left(\Gamma_{i}\right)$ is a $\Gamma_{i}$-invariant projection in $B$, we conclude that $z(\Sigma)$ is a nonzero $\Gamma$-invariant projection in $B$. Take now $g_{1}, \ldots, g_{n} \in \Gamma$ such that $\Sigma_{0}=\bigcap_{k=1}^{n} g_{k} \Sigma g_{k}^{-1}$ is finite. By definition, 
we have $z\left(g_{k} \Sigma g_{k}^{-1}\right)=\sigma_{g_{k}}(z(\Sigma))$. Since $z(\Sigma)$ is $\Gamma$-invariant, it follows that $z\left(g_{k} \Sigma g_{k}^{-1}\right)=z(\Sigma)$ for every $k$. Using (8.1), we conclude that $z(\Sigma)=z\left(\Sigma_{0}\right)$. In particular, $z\left(\Sigma_{0}\right) \neq 0$. So we find a nonzero $A$ - $\left(B \rtimes \Sigma_{0}\right)$-subbimodule $H$ of $L^{2}(p \mathcal{M})$ with $\operatorname{dim}_{B \rtimes \Sigma_{0}}(H)<\infty$. A fortiori, $H$ is an $A$ $B$-bimodule. Since $\Sigma_{0}$ is finite, also $\operatorname{dim}_{B}(H)<\infty$. Taking a projection $q \in B$ with $\operatorname{Tr}(q)<\infty$ and $H q \neq\{0\}$, it follows from Lemma 8.2 that we have found a nonzero $A$ - $B q$-subbimodule of $L^{2}(p \mathcal{M} q)$ having finite right dimension. This precisely means that $A \prec B q$ and hence, ends the proof of the theorem.

We can now deduce Proposition D.

Proof of Proposition $D$. Write $X=\mathbb{R}^{5} / \mathbb{R}_{+} \times[0,1]^{\Gamma}$ and $Y=\mathbb{R}^{5} \times[0,1]^{\Gamma}$. Put $G=\Gamma \times \mathbb{R}_{+}$ and consider the action $G \curvearrowright Y$ given by

$$
(g, \alpha) \cdot(x, y)=(\alpha \pi(g) \cdot x, g \cdot y) \quad \text { for all } g \in \Gamma, \alpha \in \mathbb{R}_{+}, x \in \mathbb{R}^{5}, y \in[0,1]^{\Gamma} .
$$

Note that the restricted action $\Gamma \curvearrowright Y$ is infinite measure preserving and can be identified with the Maharam extension of $\Gamma \curvearrowright X$. Since the Bernoulli action $\Gamma \curvearrowright[0,1]^{\Gamma}$ is mixing, we use throughout the proof that the restriction of $\Gamma \curvearrowright Y$ to a subgroup $\Lambda<\Gamma$ is ergodic whenever $\pi(\Lambda)$ acts ergodically on $\mathbb{R}^{5}$ (see e.g. [Sc82, Proposition 2.3]). Using [PV08, Lemma 5.6], we find in particular that $\Gamma \curvearrowright Y$ is ergodic, meaning that $\Gamma \curvearrowright X$ is of type $\mathrm{III}_{1}$.

Let $\mathcal{G}$ be a Polish group in Popa's class $\mathcal{U}_{\text {fin }}$, i.e. a closed subgroup of the unitary group of a $\mathrm{II}_{1}$ factor, e.g. any countable group. We claim that every measurable 1-cocycle $\omega: G \times Y \rightarrow \mathcal{G}$ is cohomologous to a continuous group homomorphism $G \rightarrow \mathcal{G}$. As explained in detail in [KS12, Step 1 of the proof of Theorem 21], it follows from [PV08, Theorem 5.3] that up to cohomology, we may assume that the restriction of $\omega$ to $\operatorname{SL}(5, \mathbb{Z})$ is a group homomorphism. By PV08, Lemma 5.6], the diagonal action $\operatorname{SL}(3, \mathbb{Z}) \curvearrowright \mathbb{R}^{3} \times \mathbb{R}^{3}$ is ergodic. It follows that the diagonal action $\Sigma \curvearrowright \mathbb{R}^{5} \times \mathbb{R}^{5}$ is ergodic as well. But then also the diagonal action $\Sigma \curvearrowright Y \times Y$ is ergodic. Since the restriction of $\omega$ to $\Sigma$ is a homomorphism and since $\Sigma$ commutes with the natural copies of $\mathbb{Z}$ and $\mathbb{R}_{+}$inside $G$, it now follows from [PV08, Lemma 5.5] that $\omega$ is also a homomorphism on $\mathbb{Z}$ and on $\mathbb{R}_{+}$. Because $\operatorname{SL}(5, \mathbb{Z}), \mathbb{Z}$ and $\mathbb{R}_{+}$together generate $G$, we have proven the claim that $\omega$ is cohomologous to a group homomorphism.

We next prove that $\mathbb{R}_{+}$is the only open normal subgroup of $G$ that acts properly on $Y$. Indeed, if $G_{0}$ is such a subgroup, we first have that $\mathbb{R}_{+} \subset G_{0}$ because $\mathbb{R}_{+}$is connected. So $G_{0}=\Gamma_{0} \times \mathbb{R}_{+}$ where $\Gamma_{0}$ is a normal subgroup of $\Gamma$ that acts properly on $Y$. Then $\pi\left(\Gamma_{0}\right)$ is a normal subgroup of SL $(5, \mathbb{Z})$. So either $\pi\left(\Gamma_{0}\right)=\{1\}$ or $\pi\left(\Gamma_{0}\right)$ has finite index in $\operatorname{SL}(5, \mathbb{Z})$. In the latter case, $\Gamma_{0}$ acts ergodically on $Y$, rather than properly. In the former case, $\Gamma_{0}$ only acts by the Bernoulli shift and the properness forces $\Gamma_{0}$ to be finite. But $\Gamma$ is an icc group, so that $\Gamma_{0}=\{e\}$.

The cocycle superrigidity of $G \curvearrowright Y$, together with the previous paragraph and [PV08, Lemma 5.10], now implies that the only actions that are stably orbit equivalent with $\Gamma \curvearrowright X$ are the induced $\Gamma^{\prime}$-actions, given an embedding of $\Gamma$ into $\Gamma^{\prime}$.

So to conclude the proof, it remains to show that $L^{\infty}(X) \rtimes \Gamma$ has a unique Cartan subalgebra up to unitary conjugacy. This follows from Theorem 8.1, using the subgroups $\operatorname{SL}(2, \mathbb{Z})<\operatorname{SL}(5, \mathbb{Z})$ (embedded in the upper left corner) and $\mathbb{Z}<\Sigma \times \mathbb{Z}$ that act recurrently on $X$ and intersect $\Sigma$ trivially.

Remark 8.3. In the formulation of Theorem 8.1, we required the existence of subgroups $\Lambda_{i}<\Gamma_{i}$ that intersect $\Sigma$ finitely and that act in a recurrent way on $(X, \mu)$. It is actually sufficient to impose the following more ergodic theoretic condition. Denote by $\Gamma \curvearrowright(Y, m)$ the (infinite measure preserving) Maharam extension of $\Gamma \curvearrowright(X, \mu)$. Consider the orbit equivalence 
relations $\mathcal{R}\left(\Gamma_{i} \curvearrowright Y\right)$ and $\mathcal{R}(\Sigma \curvearrowright Y)$, as well as their restrictions to nonnegligible subsets of $Y$. It is then sufficient to assume that for every Borel set $\mathcal{U} \subset Y$ with $0<m(\mathcal{U})<\infty$, almost

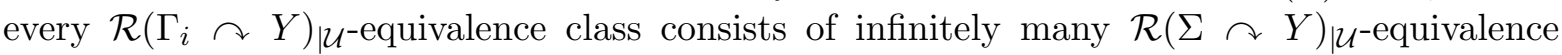
classes. Indeed, writing $B=L^{\infty}(Y)$, it then follows from [IKT08, Lemma 2.6] that for every projection $p \in B$ with $0<\operatorname{Tr}(B)<\infty$, there exist unitaries $u_{i}, v_{i} \in p\left(B \rtimes \Gamma_{i}\right) p$ satisfying 8.2 . So the proof of Theorem 8.1 goes through.

\section{References}

[BHR12] R. Boutonnet, C. Houdayer and S. Raum, Amalgamated free product type III factors with at most one Cartan subalgebra. Compos. Math. 150 (2014), 143-174.

[CH08] I. Chifan and C. Houdayer, Bass-Serre rigidity results in von Neumann algebras. Duke Math. J. 153 (2010), 23-54.

[CS11] I. Chifan and T. Sinclair, On the structural theory of $\mathrm{II}_{1}$ factors of negatively curved groups. Ann. Sci. École Norm. Sup. 46 (2013), 1-33.

[CSU11] I. Chifan, T. Sinclair and B. Udrea, On the structural theory of $\mathrm{II}_{1}$ factors of negatively curved groups, II. Adv. Math. 245 (2013), 208-236.

[DI12] I. Dabrowski and A. Ioana, Unbounded derivations, free dilations and indecomposability results for $\mathrm{II}_{1}$ factors. Preprint. arXiv:1212.6425

[Ho07] C. Houdayer, Construction of type $\mathrm{II}_{1}$ factors with prescribed countable fundamental group. J. Reine Angew Math. 634 (2009), 169-207.

[Ho09] C. Houdayer, Strongly solid group factors which are not interpolated free group factors. Math. Ann. 346 (2010), 969-989.

[HPV10] C. Houdayer, S. Popa and S. Vaes, A class of groups for which every action is $\mathrm{W}^{*}$-superrigid. Groups Geom. Dyn. 7 (2013), 577-590.

[HV12] C. Houdayer and S. Vaes, Type III factors with a unique Cartan decomposition. J. Math. Pures Appl. 100 (2013), 564-590.

[Io06] A. Ioana, Rigidity results for wreath product $\mathrm{II}_{1}$ factors. J. Funct. Anal. 252 (2007), 763-791.

[Io12] A. Ioana, Cartan subalgebras of amalgamated free product $\mathrm{II}_{1}$ factors. Preprint. arXiv: 1207.0054

[IKT08] A. Ioana, A.S. Kechris and T. Tsankov, Subequivalence relations and positive-definite functions. Groups Geom. Dyn. 3 (2009), 579-625.

[IPP05] A. Ioana, J. Peterson and S. Popa, Amalgamated free products of weakly rigid factors and calculation of their symmetry groups. Acta Math. 200 (2008), 85-153.

[KS12] J. Keersmaekers and A. Speelman, $\mathrm{II}_{1}$ factors and equivalence relations with distinct fundamental groups. Internat. J. Math. 24 (2013), Art. 1350016, 24 pp.

[Ma63] D. Maharam, Incompressible transformations. Fund. Math. 56 (1964), 35-50.

[OP07] N. Ozawa and S. Popa, On a class of $\mathrm{II}_{1}$ factors with at most one Cartan subalgebra. Ann. Math. 172 (2010), 713-749.

[OP08] N. Ozawa and S. Popa, On a class of $\mathrm{II}_{1}$ factors with at most one Cartan subalgebra, II. Amer. J. Math. 132 (2010), 841-866.

[Pi84] J-P. Pier, Amenable locally compact groups. John Wiley, New York, 1984.

[Po01] S. Popa, On a class of type $\mathrm{II}_{1}$ factors with Betti numbers invariants. Ann. of Math. 163 (2006), 809-899.

[Po03] S. Popa, Strong rigidity of $\mathrm{II}_{1}$ factors arising from malleable actions of $w$-rigid groups, I. Invent. Math. 165 (2006), 369-408.

[Po06] S. Popa, On the superrigidity of malleable actions with spectral gap. J. Amer. Math. Soc. 21 (2008), 981-1000.

[PV08] S. Popa and S. Vaes, Cocycle and orbit superrigidity for lattices in $\operatorname{SL}(n, \mathbb{R})$ acting on homogeneous spaces. In Geometry, rigidity and group actions, Eds. B. Farb and D. Fisher. The University of Chicago Press, 2011, pp. 419-451. 
[PV09] S. Popa and S. Vaes, Group measure space decomposition of $\mathrm{II}_{1}$ factors and $\mathrm{W}^{*}$-superrigidity. Invent. Math. 182 (2010), 371-417.

[PV11] S. Popa and S. Vaes, Unique Cartan decomposition for $\mathrm{II}_{1}$ factors arising from arbitrary actions of free groups. Acta Math. 212 (2014), 141-198.

[PV12] S. Popa and S. Vaes, Unique Cartan decomposition for $\mathrm{II}_{1}$ factors arising from arbitrary actions of hyperbolic groups. To appear in J. Reine Angew. Math. arXiv:1201.2824

[Sc82] K. Schmidt, Asymptotic properties of unitary representations and mixing. Proc. London Math. Soc. 48 (1984), 445-460.

[Ue07] Y. Ueda, Remarks on HNN extensions in operator algebras. Illinois J. Math. 52 (2008), 705-725. 